

\section{(2) $\mathrm{OCDE}$ \\ No 4 \\ JUIN 1963}

Publication bi-mestrielle en anglais et en français éditée par L'ORGANISATION DE COOPÉRATION ET DE DÉVELOPPEMENT ÉCONOMIQUES.

BUREAUX DE LA RÉDACTION :

Service d'Information de I'O.C.D.E., Château de la Muette, Paris $\left(16^{\mathrm{e}}\right)$.

Les articles sans copyright peuvent être reproduits à condition d'être accompagnés de la mention "Reproduit de l'Observateur de I'O.C.D.E. " en précisant la date du numéro. Deux exemplaires justificatifs devront être envoyés au rédacteur en chef. Les articles signés ne pourront être reproduits qu'avec la signature de leur auteur.

L'Organisation n'est pas tenue de rendre les manuscrits qu'elle n'a pas sollicités.

Les articles signés expriment l'opinion de leur auteurs et non pas nécessairement celle de l'O.C.D.E.

Abonnement annuel : F 10,00; FS 10,00; DM 8,3; 15-; S 2,50 .

Le numéro : F 2,00; FS 2,00; DM 1,70; 3-; \$ 0,50.

RÉDACTEUR EN CHEF : Anker Randsholt.

Toute la correspondance doit être adressée au rédacteur en chef.

PHOTOS : Page 3 : O.C.D.E.; Page 7 : B.I.R.D.; Page 8 : A.D.N.; Page 10 : Yan-Agence Rapho; Page 16 : C.E.A. - J.P. Sudre; Page 17 : General Electric, E.U.; Page 18 : C.E.A.; Page 19: I.B.M.; Page 23 Beretty - O.C.D.E.; Page 25 : O.C.D.E.; Page 27 : Robert Mottar O.C.D.E.; Page 28 : Otto Karminsky - O.C.D.E.; Pages 32-35 : Robert Mottar, College of Aeronautics, Jouan, Max-Planck Institut; U.S.I.S., Almasy; Page 39 : Louis Falquet.

\section{SOMMAIRE}

\author{
3 PER JACOBSSON
}

4 AMÉLIORATION DES CONDITIONS FINANCIËRES DE L'AIDE

Des conditions plus libérales pour les pays en développement

8 LES PAYS DE L'EST ACCROISSENT LEURS FLOTTES MARCHANDES

Concurrence accrue pour le commerce maritime

10 L'ÉTABLISSEMENT DE PLANS DE CROISSANCE : L'EXPÉRIENCE DE LA FRANCE

Les objectifs, méthodes de préparation et d'exécution du Plan français

14 L'O.C.D.E. ET LA SÉCURITÉ NUCLÉAIRE EN EUROPE

Règles internationales de sécurité

1910000 PAGES PAR AN

Les activités statistiques de l'O.C.D.E.

20 LES PUBLICATIONS STATISTIQUES DE L'O.C.D.E.

25 COMMENT ÉVITER UN RALENTISSEMENT DU PROGRES ÉCONOMIQUE

L'action de I'O.C.D.E. pour parer au manque de personnel scientifique et technique

30 LES RÈGLEMENTS ADMINISTRATIFS ET TECHNIQUES OPPOSENT AUX ÉCHANGES DES OBSTACLES PEU CONNUS

Droits de douane et limitations quantitatives ne sont pas les seuls obstacles

32 ADAPTATION DU TRAVAIL A L'HOMME

L'ergonomie illustrée

36 LE PROBLÈME DE L'ÉNERGIE EN EUROPE : PÉNURIE OU EXCÉDENT? Evolution des problèmes de l'énergie 


\section{Per Jacobsson}

L La disparition de Per Jacobsson affecte douloureusement tous ceux qui ont eu l'occasion de collaborer avec lui dans le cadre des différentes organisations internationales. Son abord chaleureux et le rayonnement de sa personnalité s'imposaient. Il savait établir avec chaque interlocuteur et chaque audience un contact direct et lui donner le spectacle réconfortant de sa vitalité et de son optimisme foncier.

Per Jacobsson a été un grand fonctionnaire international. Il avait longtemps mis son expérience au service de la Banque des Règlements Internationaux. Le Fonds Monétaire International connut, lorsqu'il y fut élu, un nouveau développement de ses activités. Il a contribué pour une part essentielle aux progrès réalisés ces dernières années dans le domaine monétaire international.

Les importantes fonctions de Per Jacobsson ont été étroitement liées à l'histoire et à l'évolution de notre Organisation. Lorsqu'il était encore à Bâle, il fut, à l'époque où se créait l'Union Européenne des Paiements, et où les difficultés rencontrées par certains pays Membres pouvaient en menacer le succès, l'un des experts auxquels fit appel l'O.E.C.E. A la tête du Fonds Monétaire International, il a fait beaucoup pour maintenir avec l'O.E.C.E., puis l'O.C.D.E., une coopération étroite qui s'est manifestée en de nombreuses occasions : plusieurs de nos pays Membres ont reçu, en des circonstances difficiles, l'aide conjointe des deux institutions.

$\mathrm{Il}$ avait bien voulu venir assister à notre dernier Conseil ministériel. A cette occasion, il avait une fois de plus affirmé sa confiance dans la coopération économique et monétaire du monde occidental.

Jean Cottier, Secrétaire Général adjoint de l'O.C.D.E.
Per Jacobsson, au Chatean de la Muette, au Conseil Ministériel de l'O.C.D.E., le 27 novembre 1962.

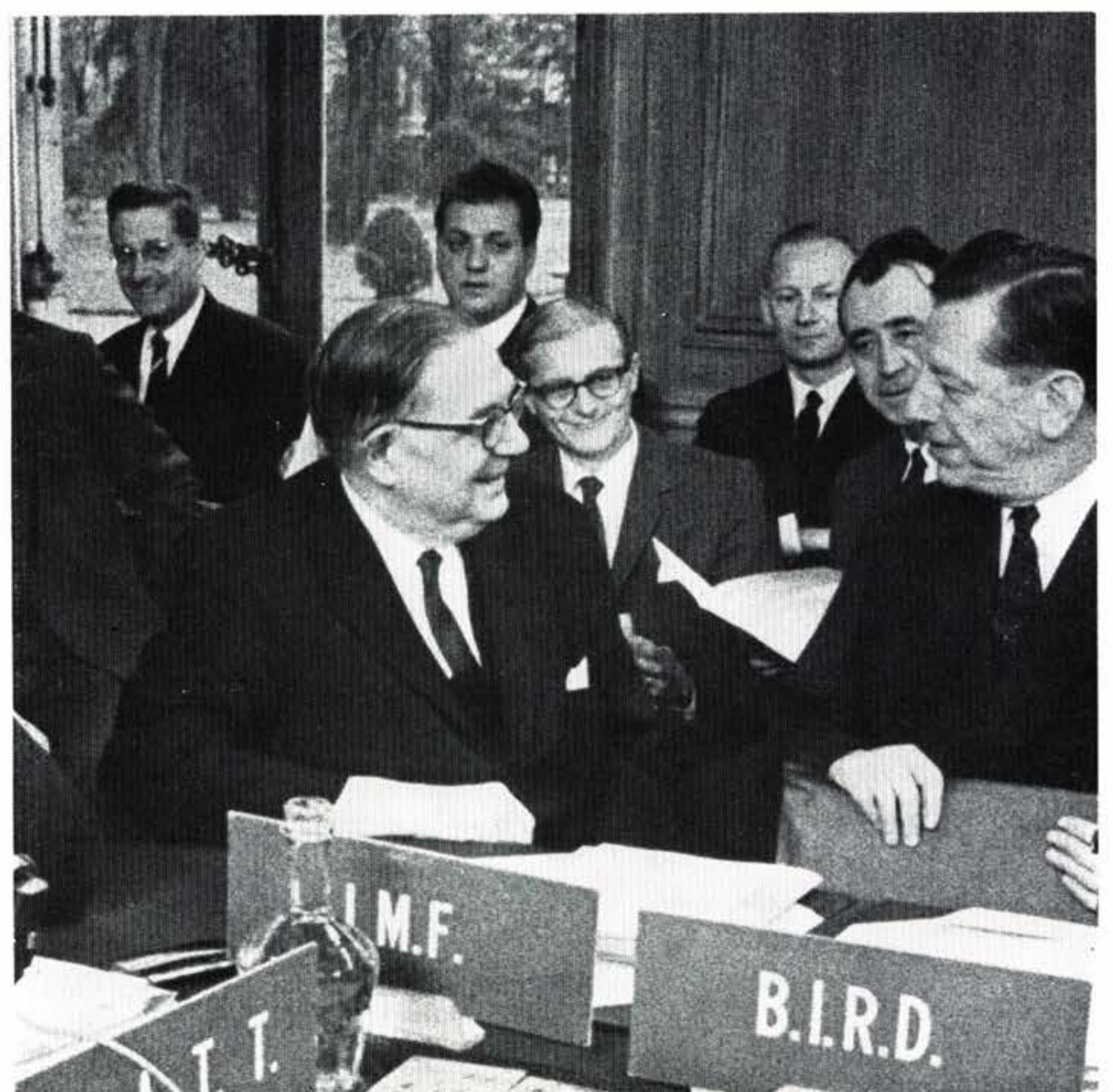




\section{amélioration des}

conditions financières

\section{de}

\section{l'aide}

Cet article a été écrit par

E.C. PARSONS

Chef de la Division du Développement Économique, Département du Développement de l'O.C.D.E.
Les activités déployées au cours des dernières années dans le domaine de l'aide à l'étranger ont fait porter l'accent, assez naturellement d'ailleurs, sur l'accroissement des moyens fournis par les pays industrialisés aux pays moins développés. Ceux-ci ne peuvent en effet opérer des investissements productifs ni bâtir ou améliorer leur infrastructure sociale et économique sans le secours de capitaux étrangers, et leurs besoins dans ce domaine sont considérables et ne cessent de croître. On a donc eu tendance à mesurer les efforts accomplis à cet égard par les divers pays industrialisés en fonction du volume de l'aide qu'ils ont apportée aux nations moins bien nanties. Il est indéniable que des progrès très nets ont été réalisés dans cette voie. C'est ainsi que le Comité d'Aide au Développement (1) de l'O.C.D.E., qui groupe les principaux pays pourvoyeurs d'aide au développement, en dehors du bloc sino-soviétique, a pu signaler que, de 1956 à 1961, le montant de l'aide bilatérale s'est accrue de quelque $75 \%$; cette progression a même atteint $23 \%$ pour le seul exercice 1960-61.

L'attention de nombreux observateurs se tourne toutefois de plus en plus vers les conditions auxquelles cette aide est accordée. Ils semblent craindre, en effet, que de nombreux pays moins développés ne s'endettent au-delà de leurs moyens en continuant d'absorber des sommes croissantes de capitaux étrangers aux conditions actuelles, et qu'ils ne s'exposent ainsi à de graves difficultés financières. C'est pourquoi le C.A.D. étudie depuis plusieurs mois les mesures qui pourraient être prises par ses membres pour essayer de résoudre ce problème.

D ès sa création, le C.A.D. a pris conscience des problèmes que posent les liens étroits qui existent entre le volume de l'aide et les conditions auxquelles celle-ci est octroyée. La Résolution sur l'effort commun d'aide, adoptée en mars 1961 et qui constitue la pierre angulaire des activités du Comité, ne préconisait pas seulement l'expansion du volume global des ressources mises à la disposition des pays moins développés et l'accroissement de l'efficacité de l'aide au développement, mais recommandait aussi qu'une place plus grande soit faite à l'assistance sous forme de dons ou de prêts assortis de conditions favorables. Le rapport du Président du C.A.D., publié en septembre 1962 (2), comprenait entre autres la recommandation suivante : «Dans la fixation des conditions financières de l'aide, il conviendrait de prêter attention à la situation et aux besoins globaux des pays bénéficiaires, tout en reconnaissant qu'aucune forme d'aide ne possède une primauté intrinsèque. "

(1) Sont membres du C.A.D., l'Allemagne, la Belgique, le Canada, le Danemark, les États-Unis, la France, le Japon, l’Italie, la Norvège, les Pays-Bas, le Portugal, le Royaume-Uni ainsi que la Commission de la C.E.E.

(2) « Les politiques et les efforts d'aide au développement en 1961 », publié par I'O.C.D.E. 
On pose souvent la question sous la forme d'une simple alternative : faut-il fournir l'aide à des conditions "classiques" ou à des conditions "libérales". Or, dans la pratique, les conditions financières dont est assorti l'octroi de fonds aux pays moins développés présentent une très grande diversité, allant du don pur et simple au crédit franchement commercial, en passant par le prêt à long terme à des conditions de faveur. Il est donc bien difficile de délimiter strictement ces diverses catégories d'opérations. Il convient de noter, en outre, que les conditions qui peuvent apparaître "libérales" aux yeux du prêteur, dans la mesure où celui-ci sait que ses propres emprunts lui coûtent plus cher, peuvent passer pour "classiques", et donc plus rigoureuses, du point de vue de l'emprunteur qui se voit contraint de payer des intérêts assez substantiels et de respecter des délais de remboursement trop courts à son gré. Il serait donc peut-être plus utile d'examiner d'abord la nature du problème et de voir ensuite comment on pourrait le résoudre.

S, il est déjà difficile d'évaluer avec précision la charge actuelle du service de la dette des pays moins développés, on ne peut en tout état de cause déterminer leur aptitude à endosser des obligations supplémentaires sans faire intervenir toute une gamme de facteurs d'ordre politique aussi bien qu'économique. On estime en gros que la dette publique extérieure (et, par conséquent, le service annuel de cette dette sous forme d'intérêts et de remboursements) a plus que doublé entre 1955 et 1961 pour l'ensemble des pays moins développés. Certains d'entre eux s'en trouvent évidemment plus affectés que d'autres. On a toutefois la certitude qu'un nombre croissant d'entre eux entrent dans une période au cours de laquelle le service de la dette absorbera probablement, chaque année, une assez forte proportion (dans certains cas, plus de $10 \%$ ) de leurs recettes d'exportation. En revanche, la position extérieure de certains autres pays reste relativement saine, dans la mesure où ils disposent de réserves de change confortables et où leur endettement se situe à un niveau raisonnable par rapport à leurs recettes d'exportation. Ces derniers pays sont donc en mesure d'emprunter à des conditions plus ou moins commerciales les fonds publics extérieurs qui leur sont nécessaires, ou même de satisfaire leurs besoins en capitaux en encourageant les investissements privés étrangers.

Il est absolument normal qu'un pays accroisse sa dette extérieure lorsqu'il entreprend un programme de développement intensif. C'est là un phénomène qu'ont d'ailleurs connu la plupart des pays durant les premiers stades de leur développement. La situation actuelle est néanmoins préoccupante en ce sens que, dans de nombreux cas, l'échéance de la dette est relativement courte et les taux d'intérêts relativement élevés. Certes, lorsque l'injection de capitaux étrangers dans l'économie d'un pays permet à celui-ci d'accroître rapidement ses exportations ou de supprimer certaines de ses importations, il peut de la sorte se procurer les devises nécessaires au service de la dette. Mais la difficulté réside dans le fait que nombre des investissements nouveaux ne sont pas immédiatement rentables et que les échéances extérieures arrivent avant que le pays ait pu gagner ou économiser un montant supplémentaire de devises suffisant pour faire face à ces obligations.

Certains pays moins développés se trouvent également aux prises avec des difficultés budgétaires dues à l'importance des sommes qu'ils doivent dégager chaque année pour assurer le service de la dette. Il leur est particulièrement difficile, en effet, de peser sur le nivcau déjà extrêmement bas de la consommation pour trouver, en plus des ressources nécessaires aux nouveaux investissements en capital, les fonds qu'exige le service de la dette.

1 faut signaler le problème particulier qui se pose à propos des crédits de fournisseurs accordés pour financer les exportations en provenance des pays industrialisés. Ces crédits, qui sont assortis de taux d'intérêt commerciaux, sont généralement remboursables dans un délai de un à cinq ans (parfois davantage pour certaines catégories de biens d'équipement, comme les avions). Bien qu'ils représentent une charge relativement lourde pour les pays intéressés, il ressort des renseignements recueillis auprès des membres du C.A.D. que leur montant ne cesse de s'accroître. Le rôle de ces crédits est, certes, considérable puisqu'ils permettent aux pays en voie de développement d'importer sans bourse délier les biens d'équipement dont ils ont besoin. Il semble bien, cependant, qu'ils soient parfois utilisés pour financer des projets qui auraient dû normalement faire l'objet de prêts à plus long terme et assortis de conditions moins rigoureuses. Il est évidemment teritant pour un pays emprunteur, lorsqu'il ne peut obtenir d'un Gouvernement étranger des prêts à des conditions favorables, de recourir au système plus facilement accessible des crédits de fournisseurs, plutôt que d'annuler un projet ou d'en suspendre l'exécution jusqu'à ce qu'il obtienne des moyens de financement à des conditions plus libérales. Il n'en reste pas moins que l'abus de ce procédé risque d'alourdir excessivement le service de la dette et d'aboutir à des demandes de révision des conditions ou de consolidation de l'ensemble de la dette extérieure.

Avant de rechercher les moyens d'atténuer ce problème du service de la dette, il convient de souligner que les pays membres du C.A.D. ont déjà beaucoup fait pour assouplir les conditions dont sont assortis les prêts du secteur public. En effet, à concurrence de $60 \%$, l'aide bilatérale accordée en 1961 (la dernière année pour laquelle on dispose actuellement de statistiques) a revêtu la forme de dons et de contributions assimilables à des dons; celles-ci comprenaient notamment un montant substantiel de capitaux fournis par les États-Unis sous forme de prêts remboursables dans la monnaie du pays bénéficiaire, et sous forme de transferts de res- 
amélioration des

conditions financières de l'aide

sources par des ventes d'excédents agricoles (dans le cadre de la Public Law 480 des États-Unis) contre paiement dans la monnaie du pays bénéficiaire. Quant aux prêts, les chiffres du C.A.D. indiquent que les délais de remboursement s'allongent régulièrement depuis quelques années; c'est ainsi qu'en 1961 les prêts bilatéraux à échéance de plus de trente ans ont représenté environ un cinquième du montant total alors qu'ils n'en constituaient qu'une proportion insignifiante il y a seulement quelques années, et que ces délais ont encore été allongés en 1962. Il convient de noter également que nombre de pays accordent maintenant un délai de grâce assez long avant que ne commence le remboursement du capital. Enfin, la proportion de prêts accordés à des taux inférieurs à ceux du commerce a également progressé.

Certaines institutions financières internationales apportent aux pays moins développés une aide assortie de conditions libérales. La grande innovation dans ce domaine a été la création de l'Association Internationale de Développement (ou I.D.A.) qui accorde des prêts à très long terme, complétant ainsi les activités normales de la Banque Internationale pour la Reconstruction et le Développement dont les prêts n'ont généralement pas une durée supérieure à vingt-cinq ans et dont les taux d'intérêt sont fonction de ceux auxquels la Banque peut elle-même se procurer des capitaux.

\section{T} outes les possibilités d'alléger les conditions des prêts n'ont cependant pas été épuisées, et il est indispensable que les pays industrialisés fassent un nouvel effort dans ce sens afin que soit plus équitablement réparti le "fardeau» de l'aide. Certains pays, la Belgique, le Canada, la France et les Pays-Bas notamment, accordent déjà la presque totalité de leur aide sous forme de dons et de contributions assimilables à des dons. C'est aussi sous cette forme que les États-Unis fournissent la plus grande partie de leur aide. Enfin, tandis que le Royaume-Uni dose à peu près également prêts et dons, d'autres pays (tels que l'Allemagne, l'Italie, le Japon et le Portugal) préfèrent consacrer la majeure partie de leur aide à l'octroi de prêts.

On doit admettre que de nombreux pays pourvoyeurs d'aide ne peuvent pas aisément remplacer les prêts de type classique par des prêts plus libéraux ou par des dons. Dans certains cas, en effet, les fonds affectés à l'aide au développement sont obtenus par l'emprunt. et l'on comprend aisément qu'un Gouvernement hésite alors à recourir aux subventions qui permettraient de prêter ces fonds aux pays moins développés à des conditions plus libérales. Il se peut même qu'un Gouvernement se trouve contraint de choisir entre accroître son aide mais en l'assortissant de conditions classiques, ou la diminuer, mais en accordant des conditions plus libérales. Un Gouvernement qui s'efforce d'appliquer chez lui une politique financière restrictive aurait sans doute quelque difficulté à justifier devant son opinion publique l'octroi à l'étranger de prêts comparables à des subventions.

De nombreux pays préférent enfin, lorsqu'ils accordent des fonds, fiinancer des projets particuliers, facilement contrôlables, plutôt que de financer des programmes généraux de développement dont il est plus difficile d'apprécier les résultats. Le prêteur peut en effet estimer que seuls les capitaux destinés à des projets économiquement rentables seront dépensés à bon escient et qu'il est donc parfaitement fondé à fixer les conditions financières du prêt en fonction des recettes escomptées.

Il convient toutefois de noter que d'importants montants d'aide ne sont liés, en fait, à aucun projet particulier, mais qu'ils sont accordés pour financer des programmes généraux de développement ou pour soutenir le niveau de la consommation.

Le problème fondamental est donc, en réalité, de concilier les intérêts disparates des parties intéressées. On trouve, d'une part, le désir naturel des donateurs de tirer un certain profit de leurs prêts (ce qui leur permet d'ailleurs d'en accorder plus facilement de nouveaux) et de s'assurer en même temps que leurs capitaux sont utilisés rationnellement. D'autre part, un certain nombre de pays moins développés se heurtent incontestablement à des difficultés pour s'acquitter de l'ensemble des obligations contractées à la suite d'une série d'emprunts. Le C.A.D. étudie actuellement divers moyens d'arriver à une solution donnant satisfaction aux deux parties.

On a déjà utilisé une méthode, qui consiste à accorder des prêts de type classique pour certains projets de développement particuliers en prévoyant un "aménagement " éventuel de la dette si celle-ci s'avère trop lourde; l'inconvénient de ce système est qu'il risque d'inciter le débiteur à prendre ses obligations à la légère. D'autres pays pourvoyeurs d'aide s'efforcent de combiner prêts classiques, dons et prêts à des conditions libérales dans des proportions adaptées à la situation de l'emprunteur. 
Cette méthode est relativement simple lorsqu'un seul pays donateur est en cause, mais elle ne va pas sans poser un problème de coordination lorsque plusieurs pays fournissent une aide sous des formes différentes à un même pays bénéficiaire. Il existe enfin une autre méthode, utilisée par I'I.D.A., qui consiste à mettre directement à la disposition du Gouvernement du pays bénéficiaire, à des conditions libérales, des capitaux que ce Gouvernement doit ensuite prêter à des conditions commerciales aux organismes responsables de l'exécution de projets particuliers.

Il sera évidemment plus aisé de résoudre ce problème si les pays fournisseurs d'aide en capital entreprennent de concert la recherche de solutions pratiques. Au cours de sa réunion des 2 et 3 avril 1963, le C.A.D. a recommandé à ses membres de s'efforcer d'atténuer la disparité des conditions et modalités de l'aide qu'ils accordent respectivement aux pays sous-développés, ce qui, dans la pratique, équivaudrait à une libéralisation des conditions pratiquées par certains d'entre eux. Ce principe devrait s'appliquer à la fois aux programmes individuels d'assistance et aux actions concertées (par exemple, les divers consortiums financiers et groupes analogues). Les membres du C.A.D. sont également convenus d'envisager l'application de l'une ou l'autre des méthodes décrites ci-dessus pour adapter les conditions de financement des projets aux possibilités correspondant à la situation d'ensemble du pays emprunteur.

Enfin, si les pays moins développés veulent recevoir un volume accru d'aide à des conditions plus libérales, ils doivent de leur côté s'efforcer de coopérer par tous les moyens avec les pays donateurs, afin que ceux-ci aient la certitude que leurs fonds seront dépensés à bon escient, et ils doivent aussi aménager leurs propres politiques et leurs plans de manière à utiliser pleinement leurs propres ressources économiques.

Il importe, en effet, que les progrès déjà accomplis dans ce sens soient poursuivis. Il y va à la fois de l'intérêt des pays industrialisés qui fournissent l'aide et de celui des pays sous-développés qui en bénéficient.

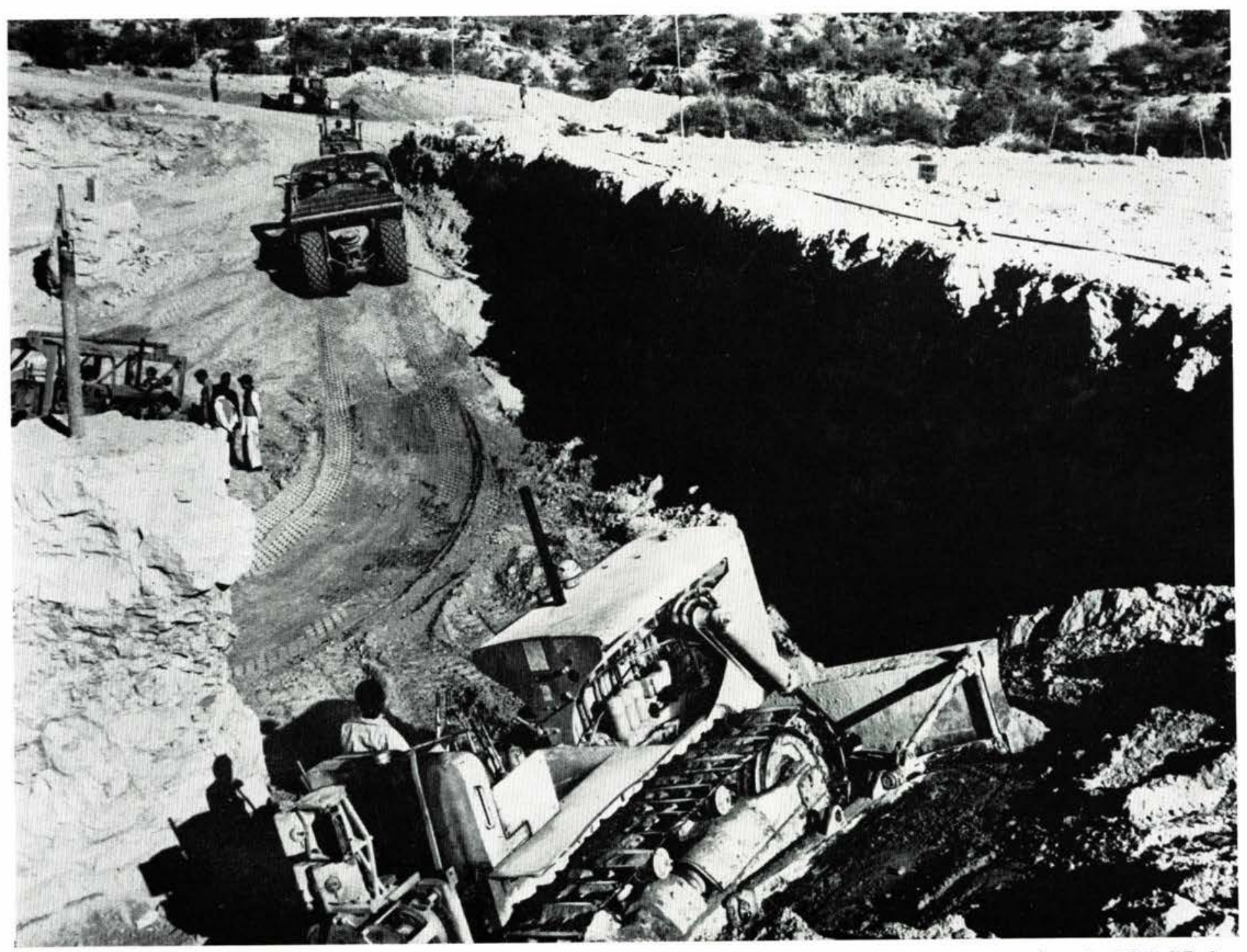

Le Traité concernant l'aménagement de l'Indus prévoit que l'Inde et le Pakistan se partageront les eaux du complexe fluvial de l'Indus. L'Allemagne, le Canada, les États-Unis et le Royaume-Uni (qui sont tous membres du Comité d'Aide au Développement), ainsi que l'Australie, la Nouvelle-Zélande, le Pakistan et la Banque mondiale, ont conclu un accord en septembre 1960 portant création d'un Fonds de Développement du Bassin de l'Indus destiné à financer les travaux d'irrigation, construction de canaux et autres aménagements en application du Traité. Le Fonds est gérê par la Banque. Ce projet est dans son genre le plus important qui ait jamais été entrepris. 

600000 tonneaux. $\mathrm{Si}$ on ajoute à ces tonnages ceux des flottes de l'Allemagne orientale, de la Bulgarie, de la Tchécoslovaquie et de la Roumanie, on atteint pour le bloc oriental un total d'environ 6,2 millions de tonneaux, dont 1.3 million de tonneaux pour les pétroliers.

Les plans que le Groupe de Travail Maritime Permanent du COMECON (Conseil d'Assistance Économique Mutuelle) a établis en vue d'accroitre les flottes du bloc oriental, menacent de faire perdre en dix ans aux pays occidentaux la quasi-totalité de ce fret étranger, qui

serait dorénavant réservé aux flottes des pays de l'Est. Ces plans prévoient une normalisation et une réduction des types de navires construits dans les chantiers, une coopération entre les pays et la suppression de toute concurrence. Le tableau donne une idée de l'ordre de grandeur du fret qui passera de ce fait dans la catégorie "restreinte ".

La principale caractéristique du commerce maritime soviétique est l'accroissement des exportations totales de pétrole. Celles-ci, qui sont passées de 18 millions de tonnes en 1958 à 20.5 millions de tonnes en 1960, devraient encore doubler d'ici 1965 , et tripler d'ici 1970 par rapport à 1960 . La flotte pétrolière actuelle de la Russie ne représente que 1,2 million de tonneaux de jauge brute; les exportations qu'elle ne peut assurer sont effectuées par des pétroliers étrangers; à eux seuls, les armateurs britanniques ont un contrat qui représente l'emploi de 16 pétroliers pendant plusieurs années.

Il paraît maintenant probable que dans les toutes prochaines années près de 2 millions de tonnes de pétroliers, parmi lesquels figureront des navires de 70000 tonnes, seront livrés à l'U.R.S.S. par des chantiers navals italiens, japonais et yougoslaves. La construction de pipe-lines entre les gisements de l'Oural-Volga et l'Europe du Nord libérera une grande partie de ce tonnage neuf de pétroliers pour le commerce pétrolier russe avec le monde occidental, Cuba et la République populaire de Chine. Pour les transports de marchandises sèches, le bloc envisage d'établir de nouvelles lignes régulières vers les pays en voie de développement d'Asie et d'Afrique; et dans les milieux maritimes, on parle de services communs, de coordination des dates de desserte et d'un tarif commun.

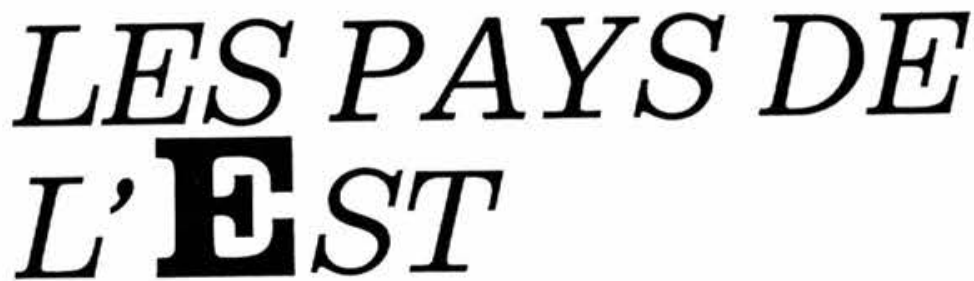

ACCROISSENT

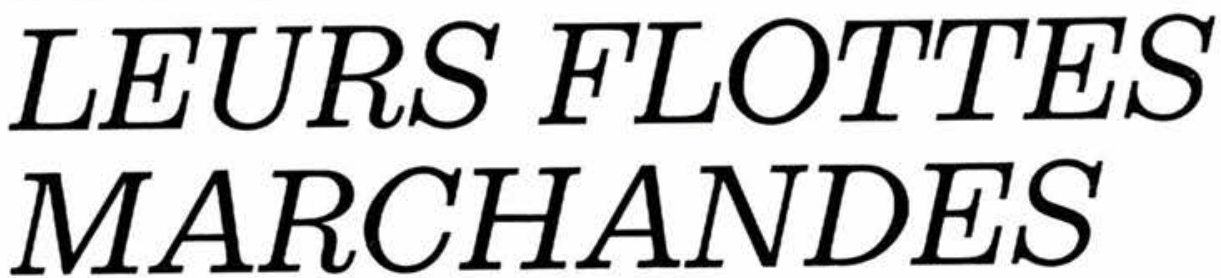

E In ce qui concerne la construction navale, I'U.R.S.S. a largement accru la capacité de ses chantiers, qui pourraient produire annuellement de 7 à 800000 tonneaux. D'ici la fin de 1965, les flottes marchandes du bloc oriental devraient atteindre une capacité totale de 10 millions de tonneaux.

Mais l'accroissement des flottes placées sous le contrôle du bloc oriental n'est pas le seul danger qui menace le commerce maritime occidental. Les

méthodes appliquées par les pays à commerce d'État, qui non seulement possèdent les navires, mais aussi achètent et vendent des produits et sont ainsi en mesure de faire varier à volonté le prix des marchandises et les taux de fret, placeront les pays traditionnels de transports maritimes dans une position extrêmement difficile, étant donné les arrangements qu'ils ont conclus en vue d'assurer la libre concurrence sur les marchés.

\begin{tabular}{|c|c|c|c|c|c|}
\hline & & \multicolumn{2}{|c|}{ Importations* } & \multicolumn{2}{|c|}{ Exportations* } \\
\hline & & 1959 & 1960 & 1959 & 1960 \\
\hline U.R.S.S. & $\begin{array}{l}\text { Cargaisons sèches } \\
\text { Pétrole }\end{array}$ & $\begin{array}{l}2,67 \\
2\end{array}$ & $\begin{array}{l}3 * * \\
2 * *\end{array}$ & $\begin{array}{l}12,14 \\
18\end{array}$ & $\begin{array}{l}13^{* *} \\
24^{* *}\end{array}$ \\
\hline POLOGNE & $\begin{array}{l}\text { Cargaisons sèches } \\
\text { Pétrole }\end{array}$ & $\begin{array}{l}7,24 \\
0,56\end{array}$ & $\begin{array}{l}9,65 \\
0,67\end{array}$ & $\begin{array}{r}10,09 \\
0,15\end{array}$ & $\begin{array}{r}11,42 \\
0,12\end{array}$ \\
\hline BULGARIE & Total & 1,71 & 1,37 & 0,87 & 0,85 \\
\hline $\begin{array}{l}\text { ZONE } \\
\text { SOVIÉTIQUE } \\
\text { D'ALLEMAGNE }\end{array}$ & Total & 2,42 & 2,80 & 1,39 & 1,67 \\
\hline
\end{tabular}

* En millions de tonnes. ** Estimations. 


\section{—L'ETABLISSEMENT DE}

Les Gouvernements des pays de l'O.C.D.E. font plus que jamais des efforts conscients et déterminés en vue d'atteindre les objectifs qu'ils se sont fixés en matière de croissance économique.

Plusieurs d'entre eux ont traduit de façon concrète cet objectif de politique économique en établissant des systèmes institutionnels en vue d'élaborer et de mettre en ouvre des plans nationaux.

Citons à cet égard le système français qui suscite un intérêt considérable.

Le présent article a été écrit par John Hackett, économiste de l'O.C.D.E. qui, avec sa femme, a écrit sur la même question un ouvrage intitulé "Economic Planning in France », devant paraître prochainement chez MM. Geo. Allen and Unwin, à Londres.

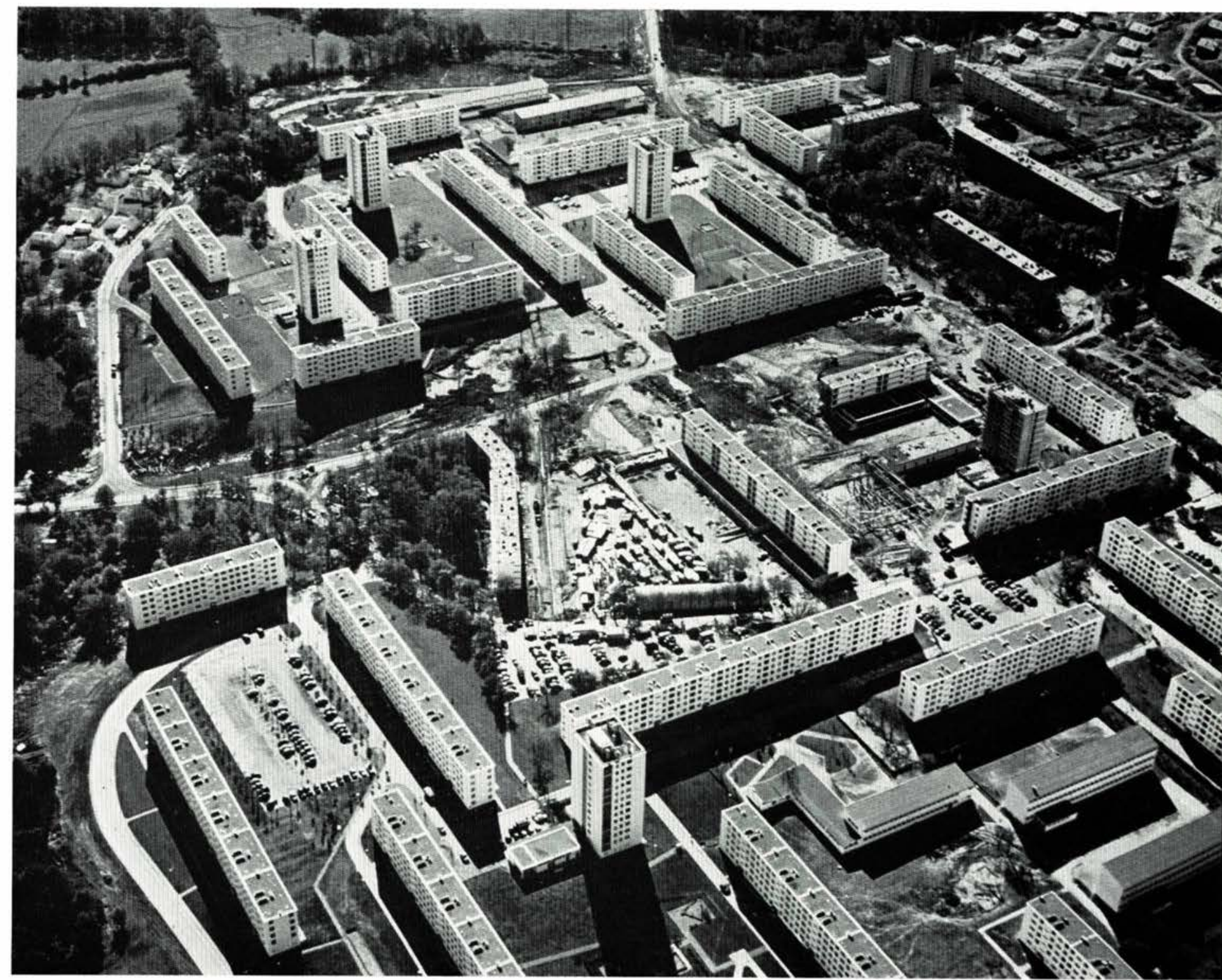

Là où s'étend aujourd'hui la ville de Mourenx, il n'y avait, voici quelques années, que des champs. La ville a été construite pour loger le personnel du complexe industriel de Lacq, dans le Sud-Ouest de la France, région au déreloppement de laquelle le 4 e Plan attache une importance particulière. 


\section{PLANS DE GROISSANGE:}

Depuis 1946, la France possède un système institutionnel de planification. Le Plan porte encore la marque de son premier architecte, Jean Monnet, mais le plan actuel - qui est le quatrième (1962-65) - diffère du premier par la complexité de ses méthodes et par la nature de ses objectifs. Le Cinquième Plan, en cours d'élaboration, constituera sans aucun doute une étape de plus dans cette évolution.

\section{Quels sont les objectifs ?}

Le Quatrième Plan est plus ambitieux à bien des égards que ses précédesseurs. Non seulement le taux de croissance retenu ( $5,5 \%$ par an) (1) est plus élevé que durant toute période équivalente depuis la guerre mais encore il doit ètre atteint dans un climat de liberté croissante du commerce extérieur et des paiements. Ceci implique, entre autres, la nécessité d'éviter un développement trop rapide de la demande, générateur de hausses de prix et de difficultés de balance des paiements. En d'autres termes, le maintien de l'équilibre économique présente une importance fondamentale pour la réussite du Quatrième Plan.

Le Quatrième Plan présente encore un certain nombre de caractéristiques nouvelles : augmentation de $50 \%$ des dépenses publiques d'ordre social, éducatif et culturel, ce qui représente l'accroissement de loin le plus rapide depuis la guerre; mise en cuvre d'une politique assurant aux revenus de certaines catégories de la population - personnes àgées, familles nombreuses, travailleurs gagnant le salaire minimum légal une croissance plus rapide que la moyenne nationale; effort pour corriger le retard dans le développement économique de certaines régions - notamment l'Ouest et le Sud-Ouest - par rapport aux plus prospères, comme la région parisienne.

\section{Elaboration du Plan}

Le trait le plus caractéristique de la planification française réside peut-ètre dans l'ampleur des consultations engagées au cours de l'élaboration du Plan auprès des organismes représentatifs des

(1) Ce chiffre s'applique à la production intérieure brule, définie selon le concept francais qui, du fait notamment qu'il exclut les services de l'Etat, diffère du concept plus usuel de produit national brut. En termes de P.N.B., lobjectif francais correspond à une croissance de $5 \%$ par an. A titre de comparaison, indiquons que, eu égard à l'objectif de $50 \%$ à alleindre en dix ans (1960-70), le taux moyen de croissance pour l'ensemble des pays de I'O.C.D.E. s'élève à $4,1 \%$. différents intérèts économiques : industrie privée, agriculture, main-d'œuvre. Les consultations s'amorcent dès le début de la préparation du Plan, au moment où on détermine les objectifs de celui-ci. Pour le Quatrième Plan, l'organisme représentatif consulté à ce stade de la procédure avait été le Conseil Économique et Social. Pour le Cinquième Plan, les discussions de politique économique seront élargies; elles inclueront un organisme représentatif d'un niveau supérieur, dénommé Conseil Supérieur du Plan (présidé par le Ministre des Finances); en outre, le Parlement aura l'occasion de discuter les objectifs généraux du Plan avant que n'en commence l'élaboration détaillée.

C'est en dernier ressort au Gouvernement qu'il incombe de choisir les objectifs, mais il a été tenu compte des avis des groupes consultés : le désir du Conseil Économique et Social de voir adopter pour le Quatrième Plan un taux élevé de croissance - $6 \%$ - n'a pas été étranger à la décision prise par le Gouvernement de fixer l'objectif à $5,5 \%$ après avoir initialement retenu $5 \%$.

Après des débats sur la politique générale à suivre, le Gouvernement communique ses décisions, sous forme d'un ensemble de directives politiques, à son organisme de planification, le Commissariat général au Plan, qui est chargé de coordonner tous les travaux techniques relatifs au Plan.

La deuxième étape de l'élaboration du Plan est d'ordre technique. Il s'agit de convertir les objectifs généraux en objectifs pour chaque secteur, et d'évaluer la possibilité de les atteindre. Ce travail est assuré par des Commissions de Modernisation, organismes tripartites où sont représentés le secteur public, les employeurs et les travailleurs. Ces Commissions sont au nombre de vingt-sept: cinq sont dites horizontales, parce qu'elles intéressent l'ensemble des branches d'activités (celle de la main-d'œuvre par exemple), vingt-deux sont verticales et traitent de certains secteurs spécialisés (sidérurgie, transports, etc.). Si l'on compte les membres des groupes de travail, quelque trois mille personnes participent aux travaux de ces Commissions. La première tâche des Commissions est de déterminer, chacune pour son secteur, les répercussions des "directives " gouvernementales sur la production, les ventes, l'investissement et l'emploi.

C'est au Commissariat général qu'il incombe de vérifier si les objectifs arrètés par les diverses Commissions de Modernisation sont compatibles entre eux et si, considérés dans leur ensemble, ils forment un tout cohérent et équilibré. A cette fin, le cadre constitué par les Comptes de la Nation apporte une aide précieuse. Enfin, il importe aussi au premier chef que les représen-
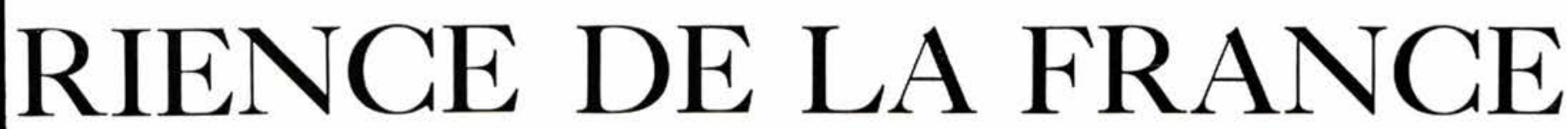


\section{L'ETABLISSEMENT DE PLANS DE CROISSANCE}

tants du secteur privé aient connaissance du cadre général de croissance adopté et du ròle qui leur est imparti.

Outre qu'elle contribue aux études statistiques, chaque Commission rédige un rapport - qui est publié - dans lequel elle analyse les problèmes et les perspectives de la branche dont elle s'occupe, à la lumière des objectifs du Plan. L'élaboration de ces rapports donne lieu à des discussions tripartites sur les problèmes communs à chaque secteur. Ces séances de "brainstorming " se sont, dans le passé, révélées extrèmement fructueuses, et ont été à l'origine de suggestions propres à améliorer le fonctionnement du secteur intéressé; bien souvent, les propositions ainsi formulées ont été entérinées par le Gouvernement, qui les a incorporées dans les textes de ses plans successifs.

Il ne faut pas se méprendre sur la nature exacte des travaux menés par les Commissions de Modernisation. Ainsi, elles ne fixent pas les objectifs de facon autoritaire. Elles n'imposent pas davantage d'objectifs particuliers à telle ou telle entreprise. Le Plan est cependant un essai d' "étude de marché généralisée " (selon le terme utilisé par le Commissaire général actuel, Pierre Massé) et permet ainsi d'étoffer et d'harmoniser les efforts déployés par les entreprises pour établir des prévisions de la demande concernant leurs propres produits.

Après une première série de consultations
- une fois encore avec le Conseil Économique et Social, puis avec le Conseil Supérieur du Plan le Plan est transmis au Parlement pour approbation et ratification. Cette approbation prend la forme d'une courte loi qui déclare en substance que le Parlement approuve le texte du Plan " comme cadre des programmes d'investissement pour la période 1962-65 et comme instrument d'orientation de l'expansion économique et du progrès social ». Le texte mème du Plan ne figure pas dans la loi, mais il lui est annexé.

\section{L'exécution du plan}

Cette exécution incombe essentiellement au Gouvernement. Celui-ci doit veiller au maintien de l'équilibre économique général et agir sur l'évolution des principales catégories de dépenses - consommation et investissements dans les secteurs privé et public — pour l'orienter dans le sens fixé par le Plan. A cette fin, lorsque le budget annuel de l'État est discuté au Parlement, il est tenu compte des progrès réalisés jusqu’à cette date dans la mise en œuvre du Plan. En outre, une procédure est prévue pour réviser chaque année les plans de production et d'investissement de chaque secteur nationalisé.

Mais les efforts du Gouvernement ne suffiraient pas à eux seuls pour assurer le succès du Plan.

Exécution des

plans :

TROISIEME PLAN 1958-1961

Au cours de la première année du Plan, des mesures de restriction s'imposaient pour arrêter l'inflation et pour rétablir l'équilibre de la balance des paiements. En fin d'année, le franc a été dévalué. Cependant, à l'expiration de la période couverte par le troisième Plan, les objectifs étaient à peu près réalisés pour la croissance globale, ils l'étaient aussi pour les investisse-
ments, mais la consommation privée restait inférieure au chiffre fixé. Les exportations et les importations avaient augmenté dans de plus fortes proportions qu'on ne l'avait prévu el l'excédent escompté pour 1961 dans la balance des paiements courants de la zone franc à l'égard du reste du monde a été réalisé dès 1959.

pourcentage d'accroissement de 1956 à 1961

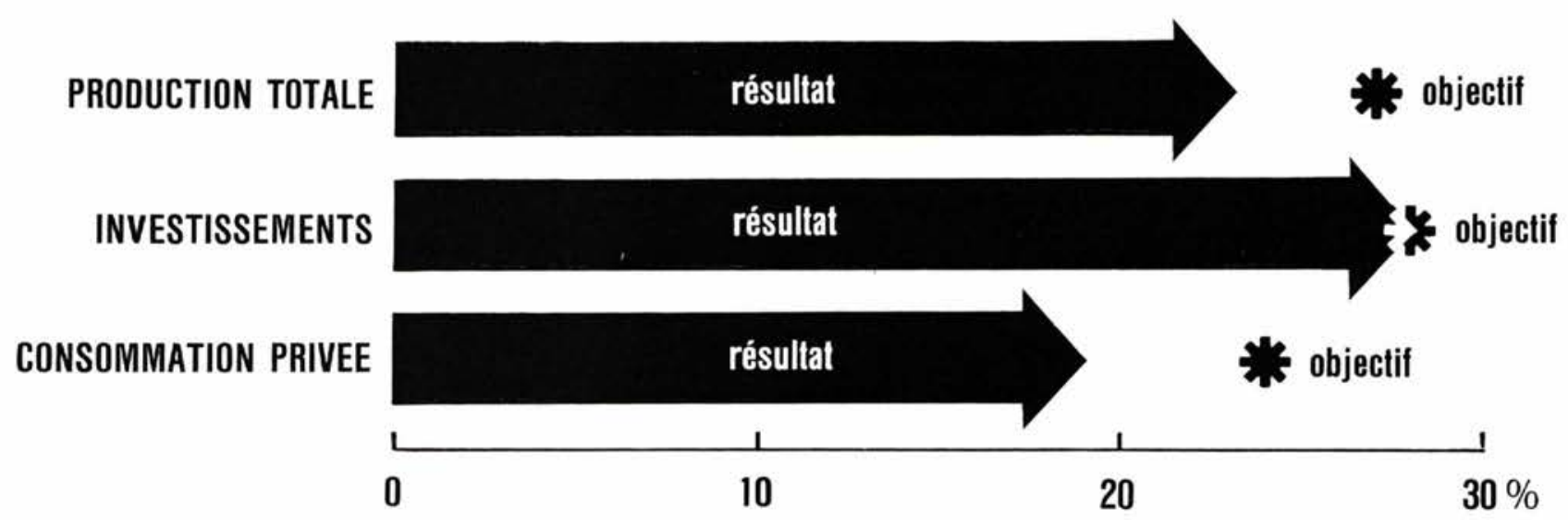




\section{L'EXPERIENCE DE LA FRANCE}

C'est ici que la participation active du secteur privé à l'élaboration du Plan prend toute sa valeur. Les milieux directement intéressés par l'exécution du Plan ayant accepté comme objectif général un taux élevé de croissance, en pleine connaissance de ses répercussions, les chances de réalisation de cet objectif en sont accrues. De plus, les divers organes consultatifs - Conseil Supérieur du Plan, Conseil Économique et Social et Commissions de Modernisation - examinent chaque année les résultats obtenus dans la mise en œuvre du Plan.

En outre, le Gouvernement dispose de plusieurs instruments, en sus du contrôle qu'il exerce sur les activités du secteur public, pour influer sur le secteur privé. Après la guerre, cette action a eu pendant un certain temps un caractère essentiellement restrictif et discriminatoire. Le Plan portait sur un petit nombre de secteurs-clés, qui bénéficiaient d'un régime préférentiel pour leurs approvisionnements en matières premières et leur financement, ce qui leur assurait une position privilégiée. Aujourd'hui, ce type d'action a beaucoup perdu de son importance. On l'utilise encore, dans une certaine mesure, pour le développement régional : de forts stimulants sont offerts aux entreprises qui vont s'établir dans les régions défavorisées, et l'expansion de l'industrie dans la région parisienne, déjà surpeuplée, est strictement contrôlée.
Pour le reste, on a essentiellement recours à des stimulants - généralement marginaux par l'intermédiaire de la politique du crédit et de la politique fiscale.

Pour la politique du crédit, étant donné que l'industrie en France, plus que dans certains autres pays, fait appel aux crédits bancaires à moyen terme, les banques d'État, qui jouent un rôle essentiel dans l'octroi de ces crédits, peuvent demander l'avis du Commissariat général au Plan lorsqu'elles reçoivent des demandes de crédit. Et le Ministère des Finances possède les moyens de limiter l'accès des entreprises privées au marché des capitaux à long terme.

Le Ministère des Finances peut aussi accorder certaines exonérations fiscales aux entreprises qui recourent au financement par l'émission d'actions sur le marché des capitaux. Il a également la possibilité d'accorder des privilèges fiscaux pour encourager le développement de la recherche scientifique, le démarrage de fabrications nouvelles ou la transformation et la reconversion d'entreprises.

Dans un nombre limité de cas, les autorités passent avec des entreprises privées des accords globaux dits "quasi-contrats": en échange de certains avantages en matière de crédits et d'impôts, ces entreprises adaptent leurs programmes de production et d'investissement aux objectifs du Plan.

\section{QUATRIEME PLAN 1962-1965}

En 1962, première année du Plan, l'objectif de production a été dépassé; il est probable qu'en 1963 la croissance sera un peu moins rapide qu'on l'avait escompté. L'augmentation des dépenses d'investissements, qui l'an dernier a été supérieure aux prévisions, se ralentira probablement en 1963. La consommation privée s'est accrue plus rapidement en 1962 qu'on ne l'avait prévu en raison notamment de l'arrivée en France de 600000 rapatriés d'A frique du Nord. Néanmoins les revenus tendent actuellement à augmenter plus rapidement que la production. Cela confirme la nécessité, soulignée dans le quatrième Plan, de mettre en auvre une politique des revenus.

pourcentages annuels d'accroissement

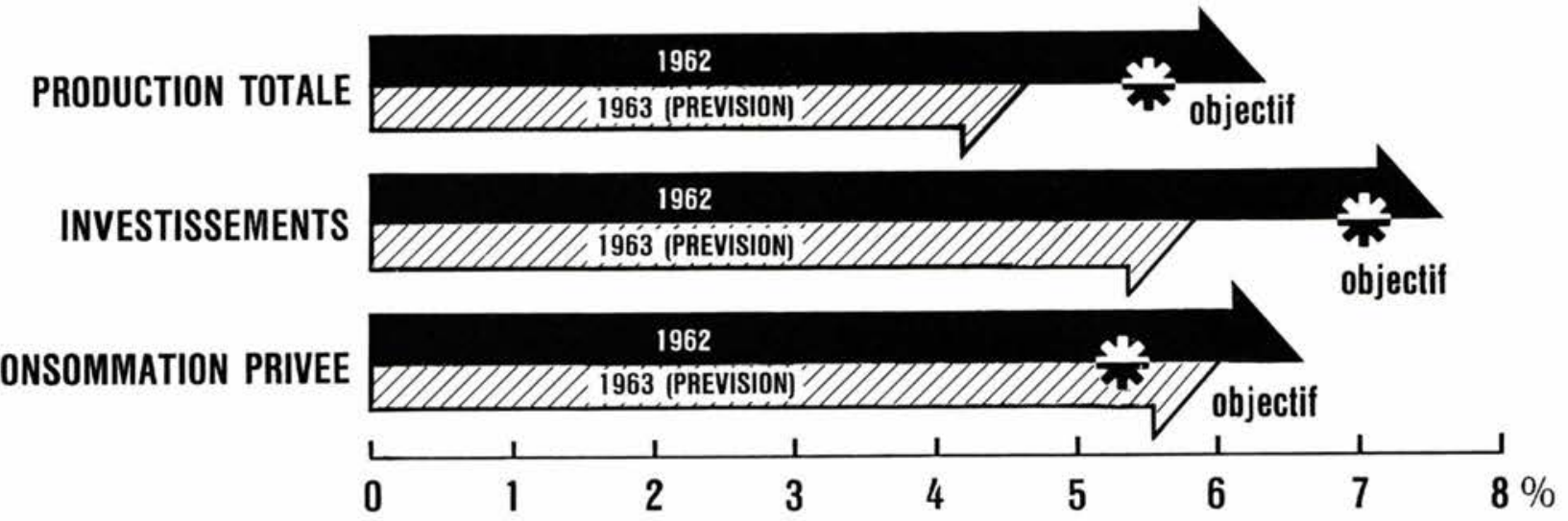




\section{L'OCDE}

L'énergie nucléaire doit apporter une contribution croissante à nos approvisionnements en énergie. Mais elle nécessite, de ce fait, la manipulation, le transport et le traitement de quantités de plus en plus importantes de substances radioactives, dont certaines trouvent des utilisations nouvelles dans la recherche, l'agriculture et l'industrie. Ces substances seraient dangereuses si des mesures rigoureuses de surveillance et de protection n'étaient adoptées, et l'une des fonctions essentielles de l'Agence Européenne pour l'Energie Nucléaire est d'étudier ces mesures pour l'ensemble des pays européens de I'O.C.D.E.
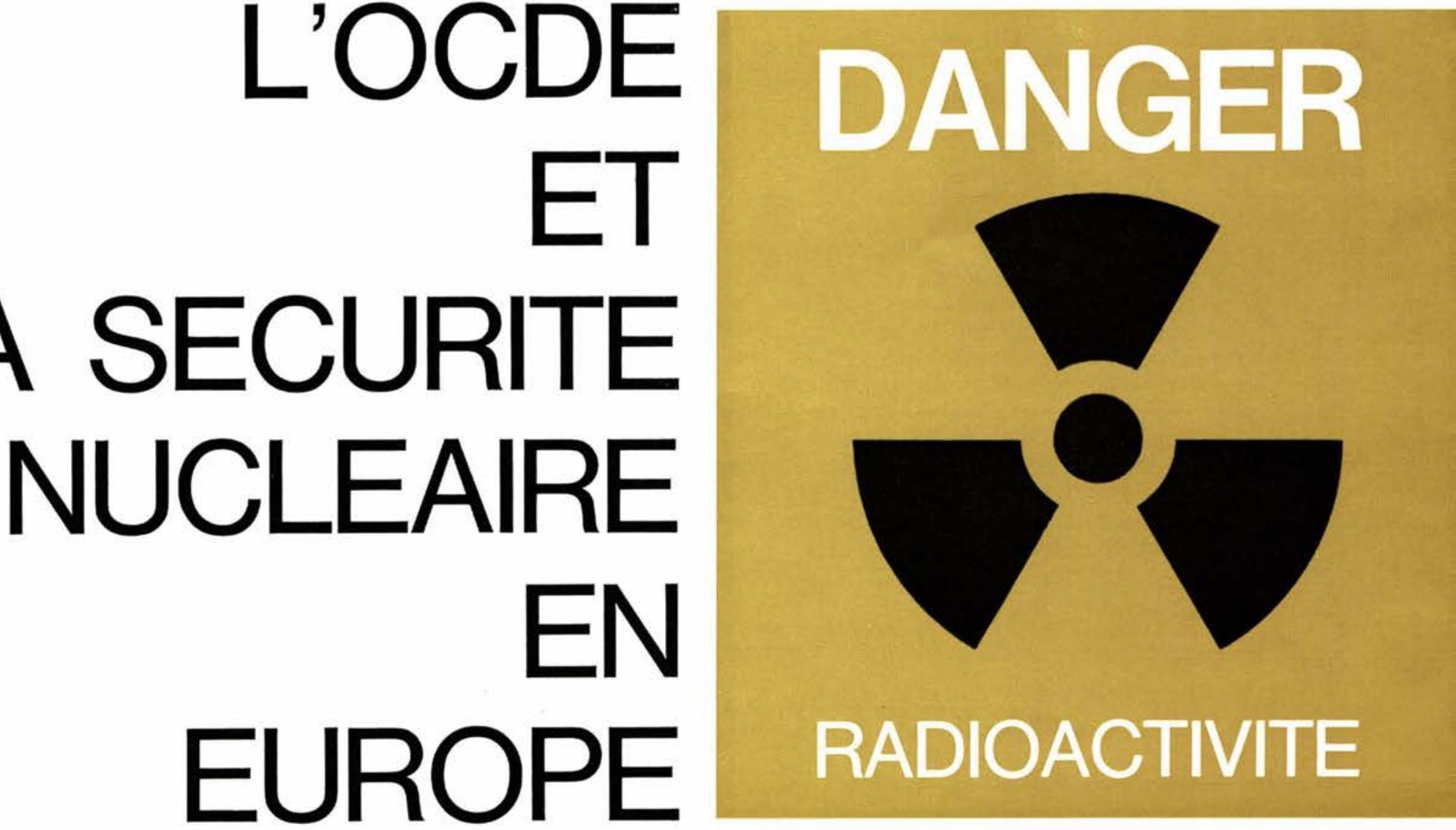

Sans doute la radio-activité est-elle largement antérieure à l'apparition même des hommes de la terre, mais c'est tout récemment seulement que l'on a pu créer artificiellement des sources capables d'émettre de grandes quantités de radiations et qui, si elles n'étaient convenablement contrôlées, pourraient devenir dangereuses pour toutes les formes de la vie. Les réacteurs nucléaires, notamment ceux des grandes centrales nucléaires qui apportent déjà une certaine contribution à nos approvisionnements en énergie, sont la principale source de production de ces substances radio-actives.

Les dangers des radiations sont dès maintenant bien connus, mais il n'est pas encore possible de prévoir exactement certains des effets à long terme (en particulier les effets génétiques) que peut entraîner une exposition aux radiations ; c'est pourquoi les autorités responsables de la mise en œuvre des programmes nucléaires nationaux ont adopté dès l'origine des dispositions très strictes de sécurité et de protection.

Enfin, les risques nucléaires ne connaissent pas de frontières et ceci vaut particulièrement pour 




\section{L'OCDE}

ET

LA SECURITE

NUCLEAIRE

EN EUROPE

méthodes de "comptabilisation des doses " et des règles ont été fixées pour assurer la protection contre des doses cumulées excessives ou les cas d'exposition anormale. On trouve également, dans la décision du Conseil, des recommandations relatives au contrôle individuel des doses reçues, aux examens médicaux et à la surveillance dans les «zones contrôlées".

Il est prévu en particulier que toute personne employée, susceptible d'être exposée aux dangers des radiations ionisantes, doit être informée des risques qui peuvent en résulter pour sa santé, des techniques de travail, des précautions à prendre ainsi que des moyens de se conformer aux règles de protection contre les radiations et aux consignes médicales. En principe, aucune personne ayant moins de dix-huit ans révolus ne peut exercer une activité professionnelle présentant un danger d'exposition à des radiations ionisantes.

Les normes adoptées en 1959 ont été révisées par le Sous-Comité de la Santé et de la Sécurité de l'ENEA, compte tenu de l'évolution des connaissances scientifiques et des travaux plus récents de l'I.C.R.P. Les normes révisées ont été adoptées par le Conseil de l'O.C.D.E. en décembre 1962.

\section{Radioactivité ambiante}

L'application pratique des normes de protection de la santé implique l'existence, dans chaque pays, d'un système de surveillance et de contrôle de la radio-activité. Ce système comprend normalement deux parties distinctes :

- des stations locales de contrôle rattachées à chaque installation nucléaire et destinées à contrôler les radiations émises ou la contamination provoquée;

- un contrôle général du niveau de la radio-activité ambiante dans son ensemble, sans relation directe avec une source particulière.

La plupart des pays ont créé ou projettent de créer un réseau de stations équipées pour effectuer ces types de mesures et l'ENEA a pris des dispositions permettant la centralisation, la comparaison et la diffusion des informations provenant de ces stations. Le Sous-Comité de la Santé et de la Sécurité de l'Agence a aussi entrepris des travaux en vue de la normalisation des méthodes de prélèvement et de mesure employées dans les différents pays.

Les travaux comportant un risque de radioactivité sont effectués en cellules chaudes.

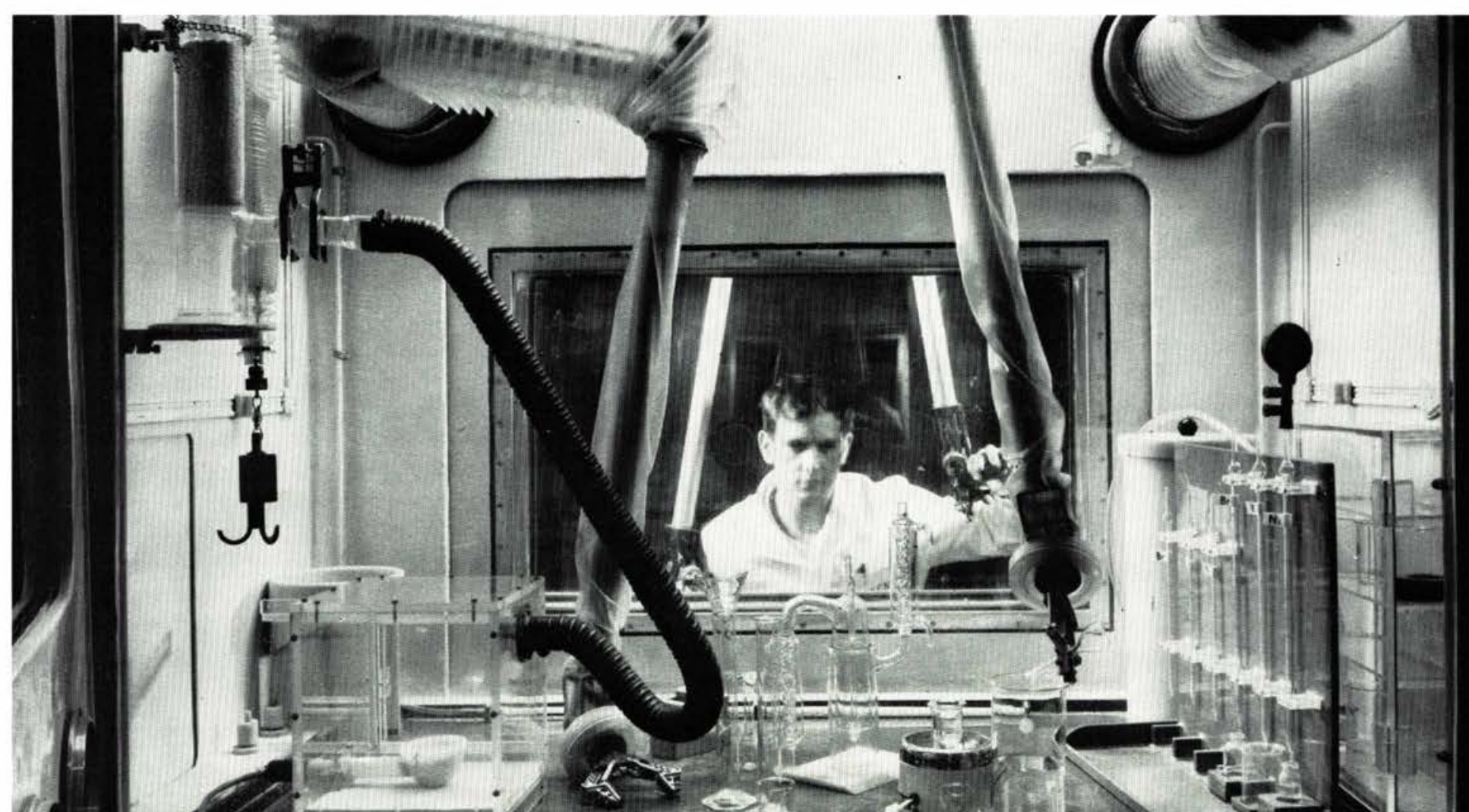




\section{Système de surveillance et d'alerte}

A la suite des dispositions prises par l'ENEA pour centraliser les résultats des mesures de la radio-activité ambiante dans les pays Membres, un système européen de surveillance et d'alerte en cas d'augmentation anormale de la radio-activité a été institué en juillet 1961. Par ce système, toute augmentation importante de la radio-activité observée dans un pays Membre est immédiatement portée à la connaissance des autorités compétentes des autres pays Membres et de l'ENEA, de sorte que toutes les mesures nécessaires de protection de la santé puissent être prises sans délai.

Pour l'application de ce système, l'augmentation de la radio-activité constatée dans un pays doit être notifiée dans les cas suivants :

- Cas d'alerte, c'est-à-dire d'un accident nucléaire ou d'une augmentation importante de la radioactivité entraînant des conséquences sanitaires régionales.

- Cas de pré-alerte, c'est-à-dire d'une augmentation importante de la radio-activité d'origine inconnue ou résultant d'accidents nucléaires mineurs et ne nécessitant pas de mesures de protection sanitaires locales.

- Cas d'intérêt scientifique, c'est-à-dire d'une augmentation anormale de la radio-activité d'intérêt purement scientifique.

Les conditions de transmission des informations diffèrent selon qu'il s'agit de l'une ou l'autre de ces éventualités; en cas d'alerte, les informations doivent être notifiées " par les moyens de transmission les plus rapides " aux autorités officiellement désignées par les Gouvernements des pays voisins.

Il y a peut-être intérêt à signaler que depuis l'établissement du système aucune information n'a eu à être transmise.

\section{Transport de matières radio-actives}

Le transport des matières radio-actives pose un certain nombre de problèmes spéciaux relatifs à la protection de la santé et à l'assurance contre les accidents. Ces problèmes présentent une grande importance pour les entreprises communes de

Dans un laboratoire de recherches sur le plutonium, les travailleurs portent des scaphandres en matière plastique pour éviter la contamination $\alpha$ à haute activité.

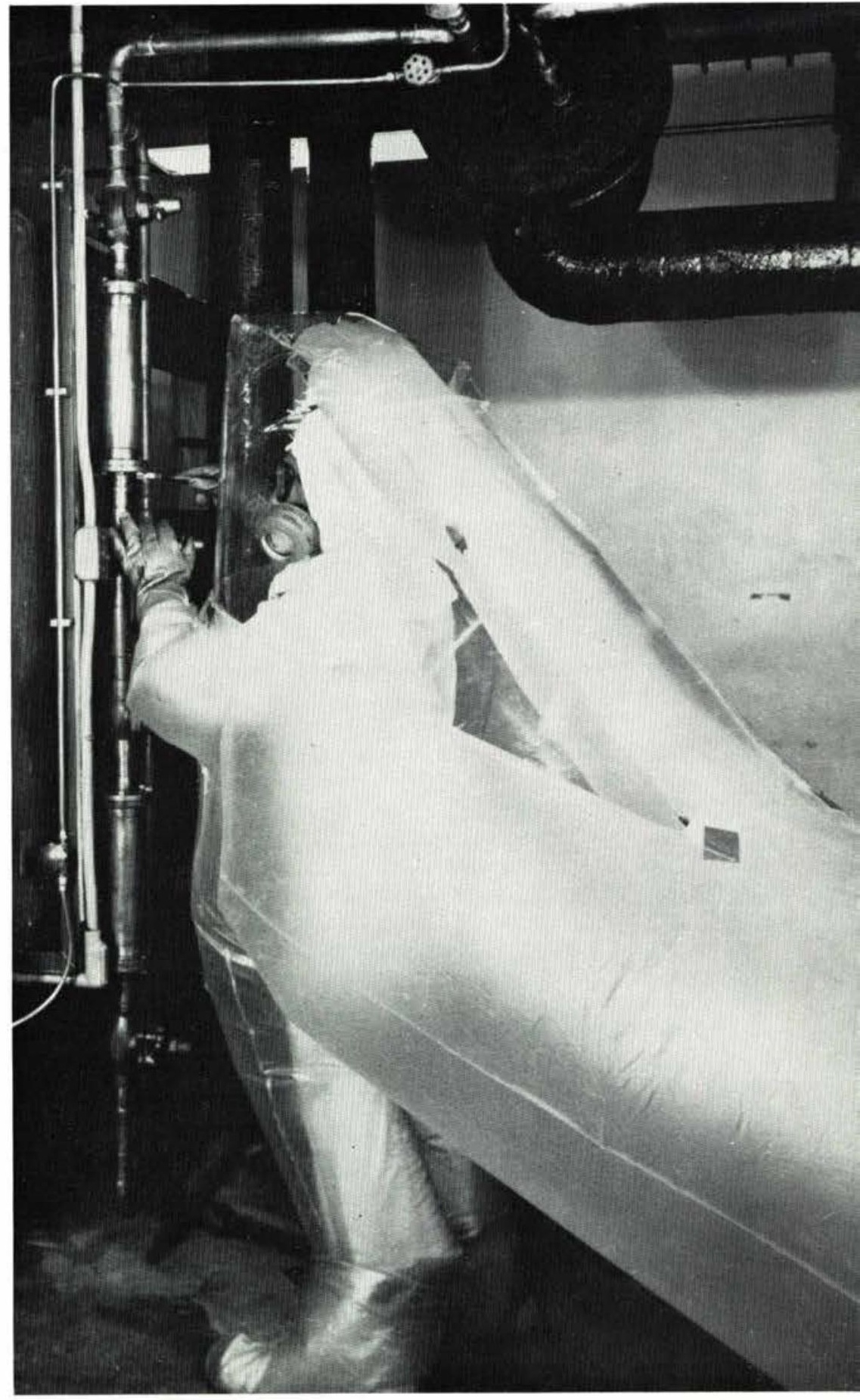




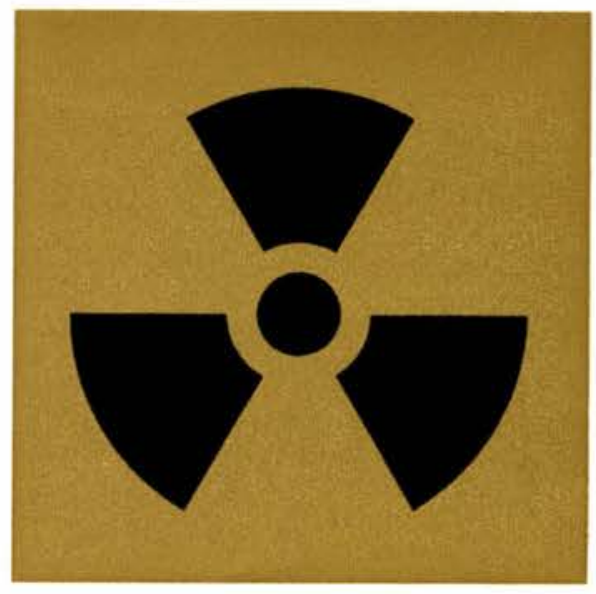

\section{L'OCDE ET}

\section{LA SECURITE} NUCLEAIRE EN EUROPE

l'ENEA - et notamment la Société européenne pour le traitement chimique des combustibles irradiés (Eurochemic) dont l'exploitation nécessite le transport de combustibles irradiés et de matières radioactives à travers de nombreux pays européens.

L'ENEA et la Société Eurochemic ont participé au cours des dernières années aux travaux qui ont abouti à l'établissement de règles internationales concernant le transport des combustibles nucléaires et des matières radio-actives, notamment aux travaux de l'Ägence Internationale de l'Énergie Atomique et du Comité International des Transports par chemins de fer.

\section{Conférences techniques et symposiums}

Les travaux de l'ENEA en matière de sécurité nucléaire en Europe ne se limitent pas à une action administrative et réglementaire. La mise au point et l'amélioration des techniques de santé et de sécurité dépendent, dans une large mesure, du progrès des connaissances scientifiques relatives aux méthodes pratiques de surveillance et de protection. C'est pourquoi l'Ägence a organisé plusieurs colloques permettant à des spécialistes venus des pays de l'O.C.D.E. d'échanger leurs informations et de confronter leurs méthodes et leurs résultats. Le premier colloque de ce type s'est tenu à Risö (Danemark) en 1959 et a été consacré aux méthodes techniques de la physique de santé dans les installations nucléaires. Un deuxième symposium sur le contrôle de la criticalité a eu lieu à Karlsruhe en 1961, et un autre symposium sur la dosimétrie individuelle s'est tenu à Madrid il y a quelques semaines. A chacune de ces réunions ont assisté environ cent experts venus des centres de recherche et des installations nucléaires d'Europe et d'Amérique du Nord.

Dans un autre ordre d'idées, l'ENEA a également organisé des conférences d'information destinées aux spécialistes des syndicats, pour leur exposer les risques que présente l'industrie nucléaire pour la santé et la sécurité, ainsi que les mesures de protection d'ordre technique et juridique prises par les pays de l'O.C.D.E.

Un pluviomètre en matière plastique'est utilisé au Centre Nucléaire de Saclay pour mesurer la radioactivité dans l'atmosphère.

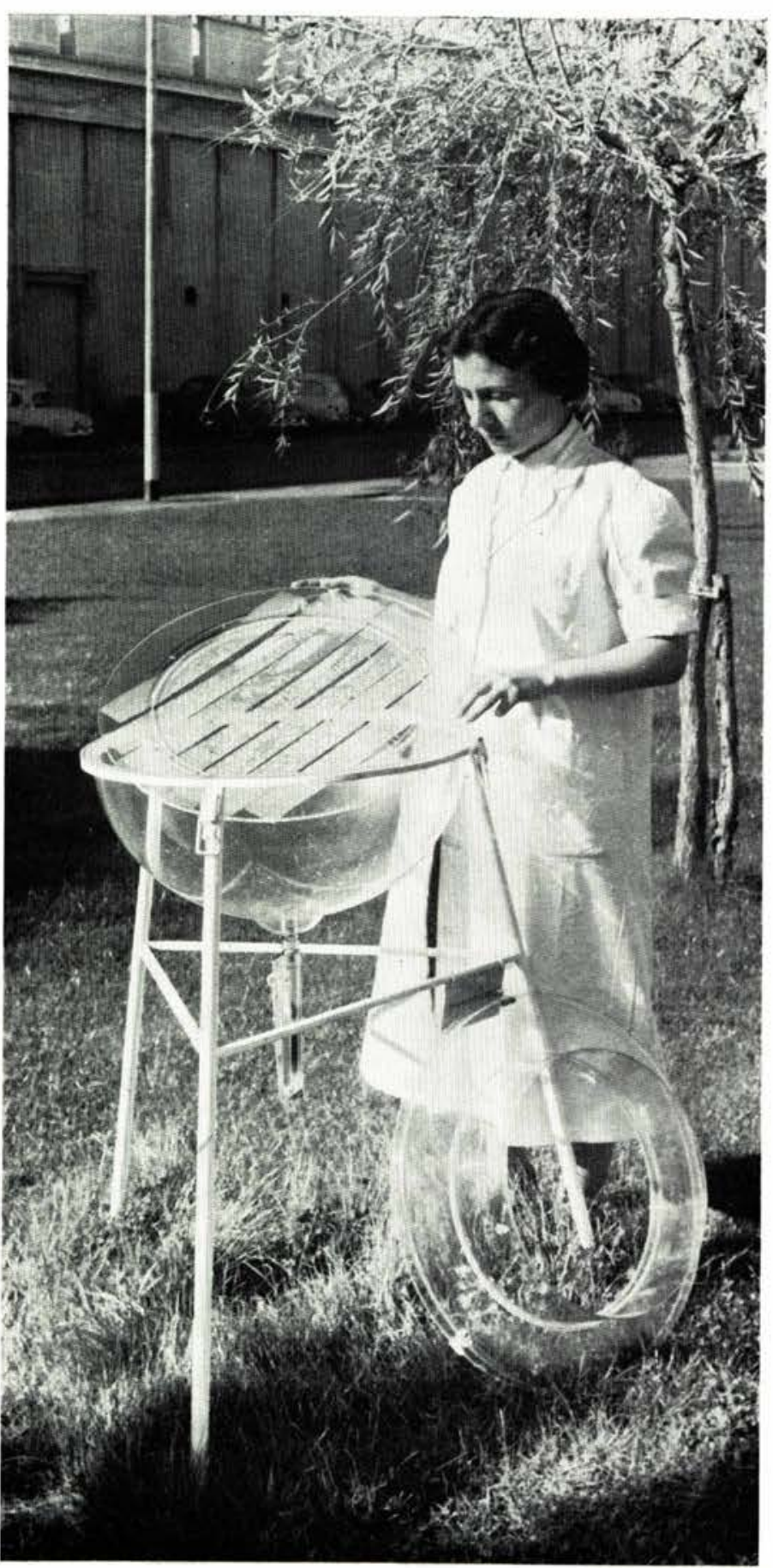




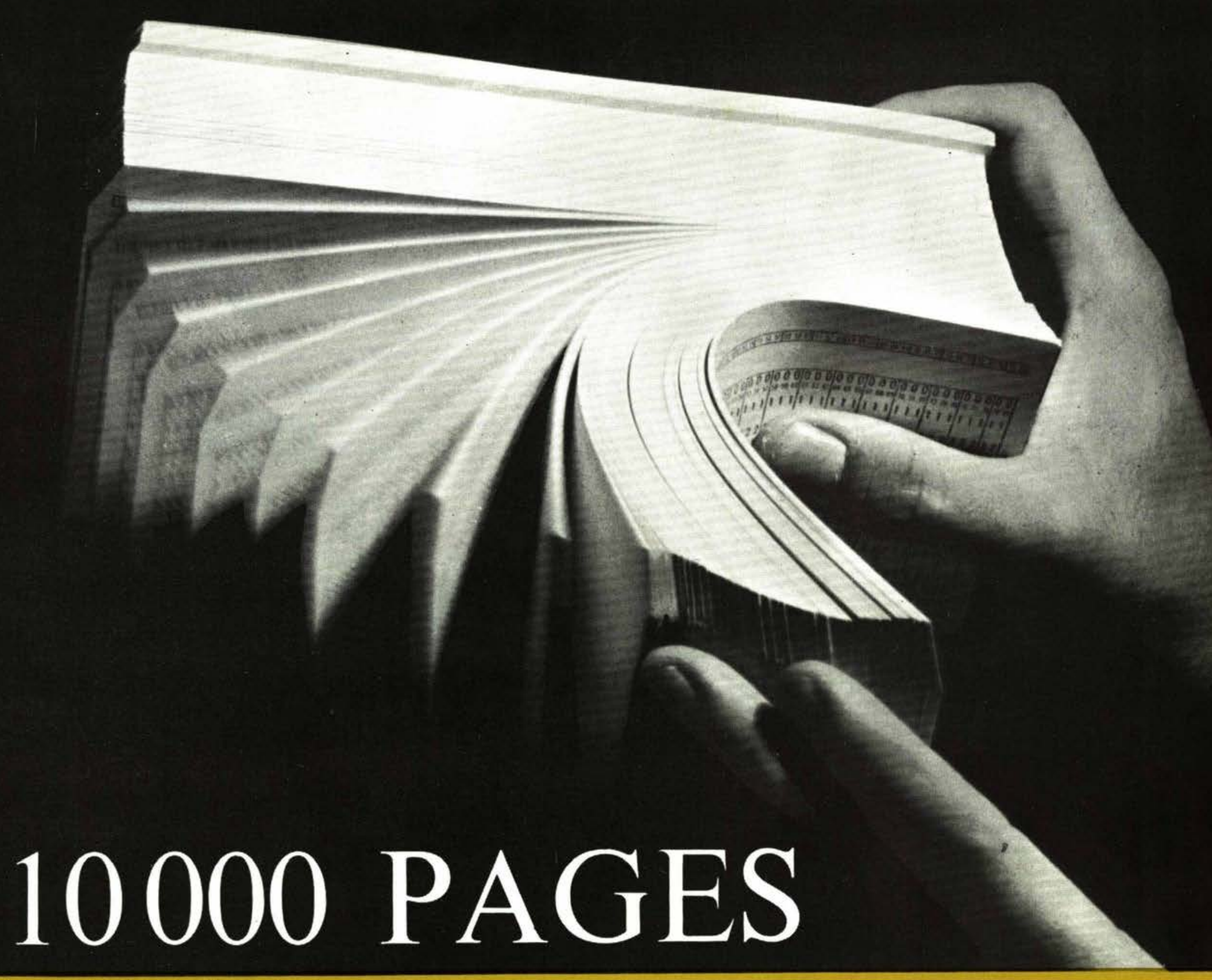

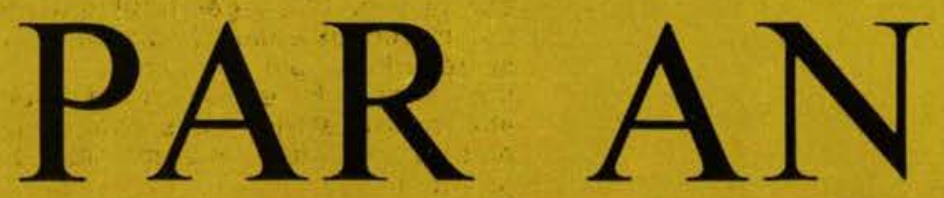

$P$ que toute l'activité de I'O.C.D.E. repose d'une façon ou d'une autre sur des chiffres. Chiffres de la production, de l'emploi, des salaires et des prix; chiffres des réserves d'or et de devises, des taux d'intérêt et des mouvements de capitaux; chiffres des importations de textiles en provenance des pays sous-développés et des exportations d'automobiles vers les États-Unis; chiffres de l'emploi dans l'agriculture et du montant des subventions versées aux exploitants agricoles; statistiques de la capacité des installations sidérurgiques, de la consommation de pétrole et du coût des centrales nucléaires; nombres d'élèves inscrits dans les écoles primaires et secondaires et chiffres des effectifs et de la demande d'ingénieurs diplômés.

On pourrait poursuivre eette énumération presque indéfiniment. Certains de ces matériaux statistiques sont confidentiels; mais la majeure partie est mise à la disposition du public. Au total, plus de 10000 pages de tableaux statistiques sont publiées chaque année.

\section{les activités statistiques} de l'OCDE

\section{D'où les chiffres proviennent-ils ?}

L'O.C.D.E. est une organisation intergouvernementale. Elle ne recueille pas elle-même les renseignements auprès de ceux qui en sont la source première : chefs d'entreprises, salariés, ménagères, etc. Ses informations proviennent d'abord des services statistiques nationaux et, dans une moindre mesure, d'instituts de recherche semipublics, d'organisations professionnelles, etc. Plus de cinquante questionnaires sont adressés chaque année aux vingt pays Membres. En outre, le Secrétariat travaille en permanence à mettre en forme les renseignements qu'il tire de centaines de journaux, de bulletins statistiques et d'annuaires rédigés en de multiples langues.

\section{Le rôle de l'O.C.D.E.}

Le rôle sans doute le plus important que joue l'O.C.D.E. dans le domaine des statistiques est aussi, 


\section{LES PUBLICATIONS STATISTIQUES DE L'OCDE}
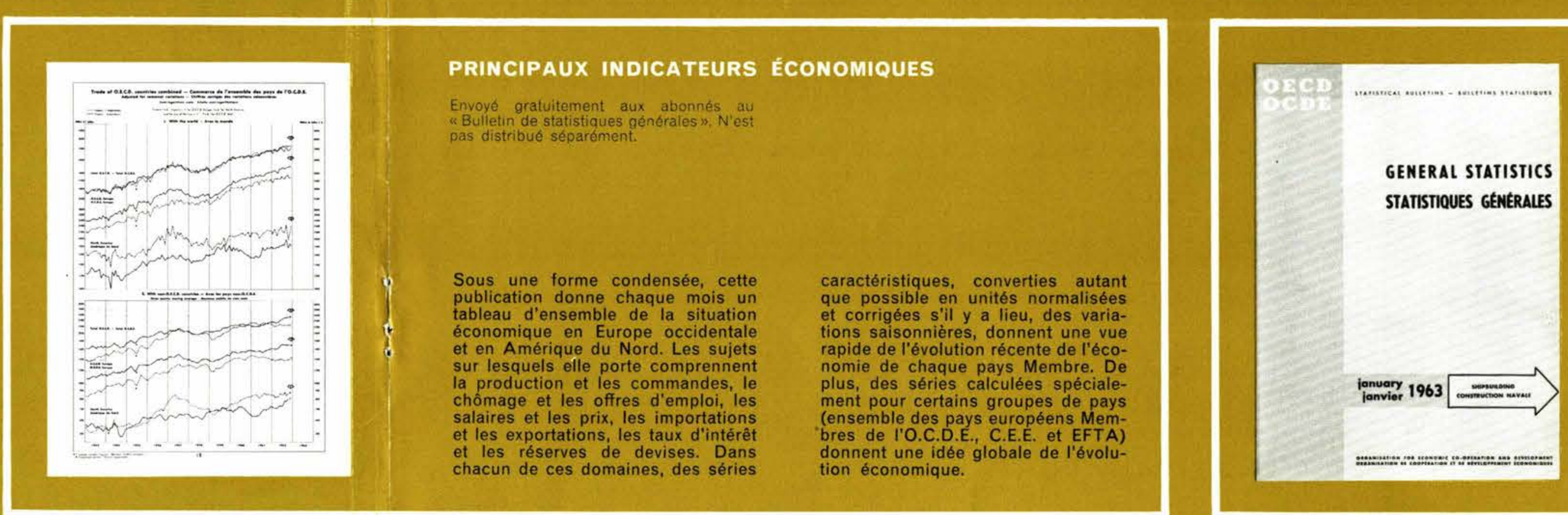

BULLETIN DE STATISTIQUES GÉNÉRALE

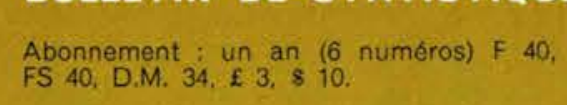
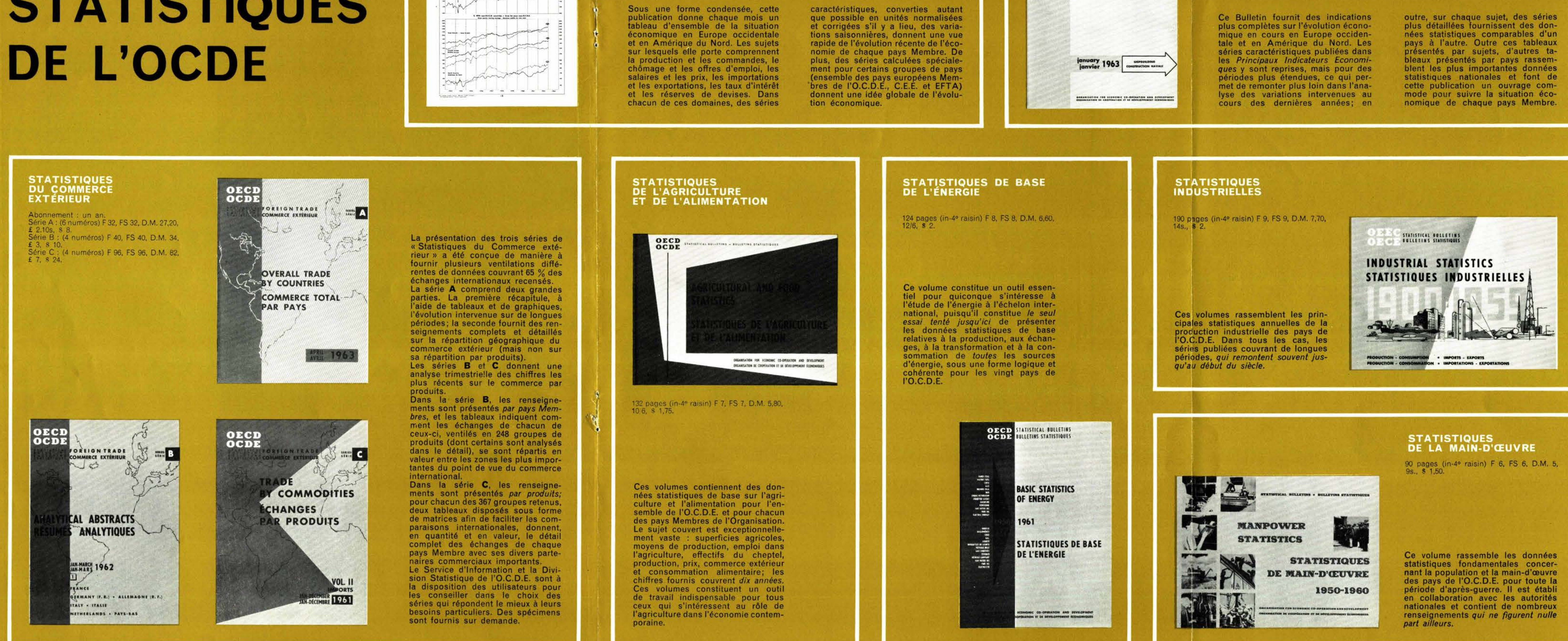

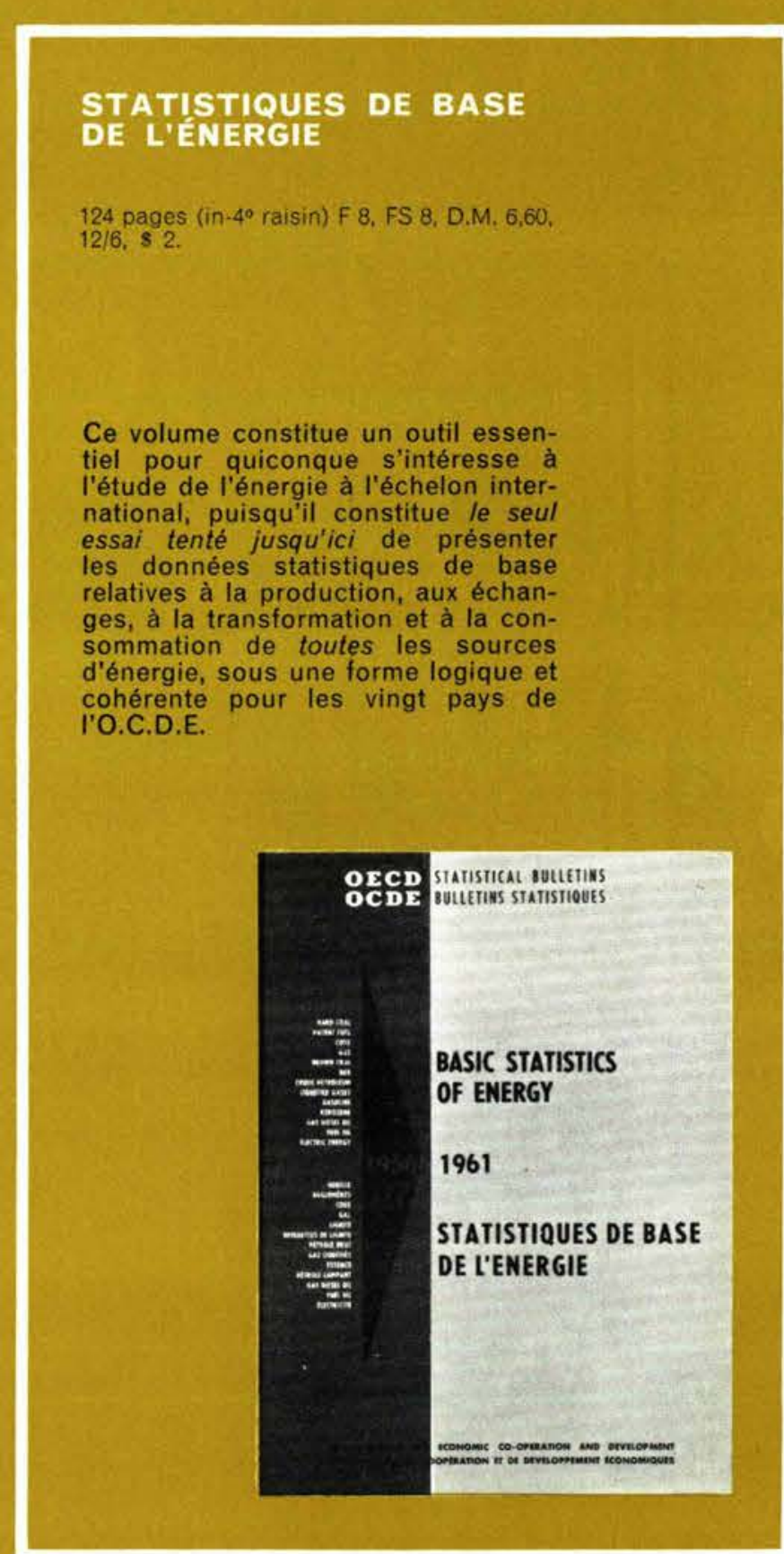

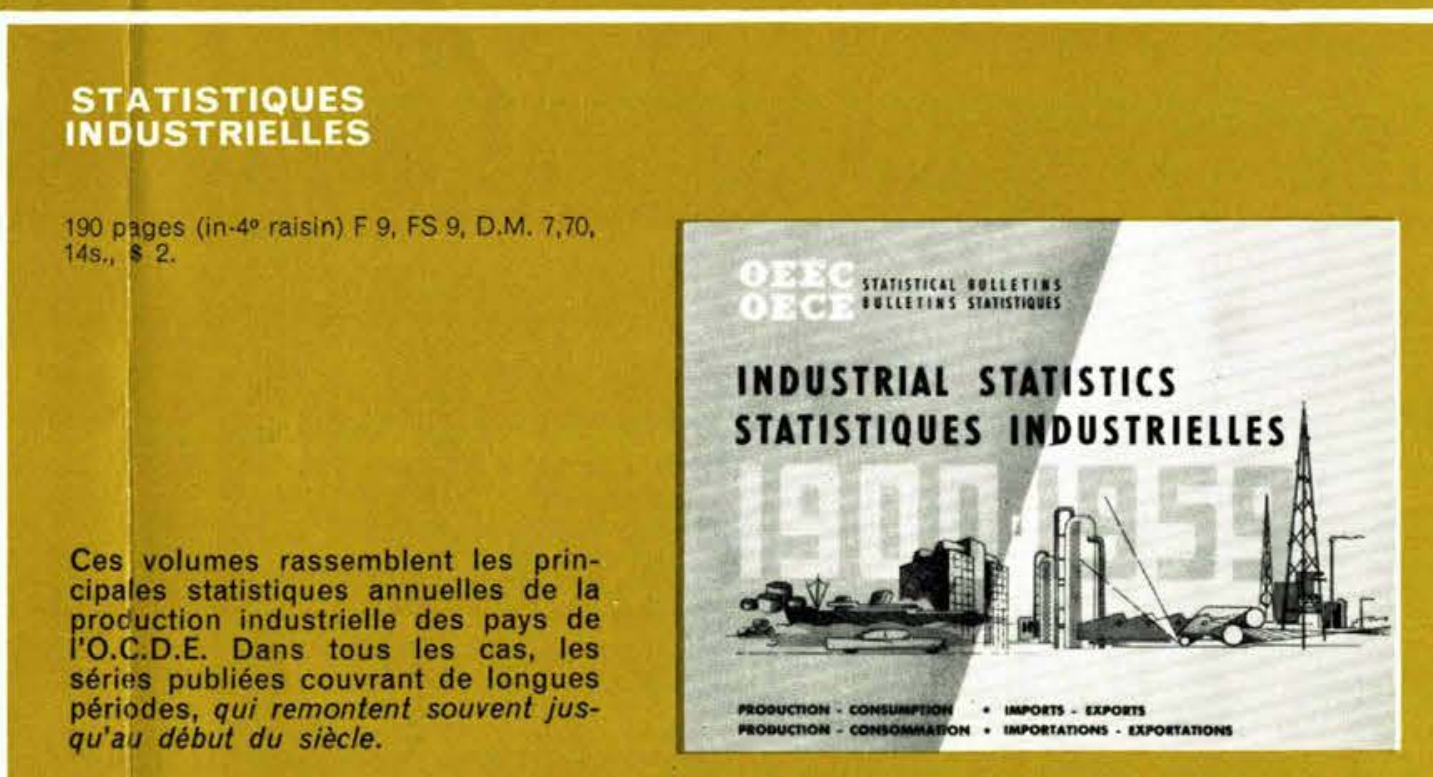

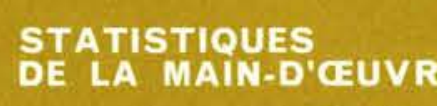

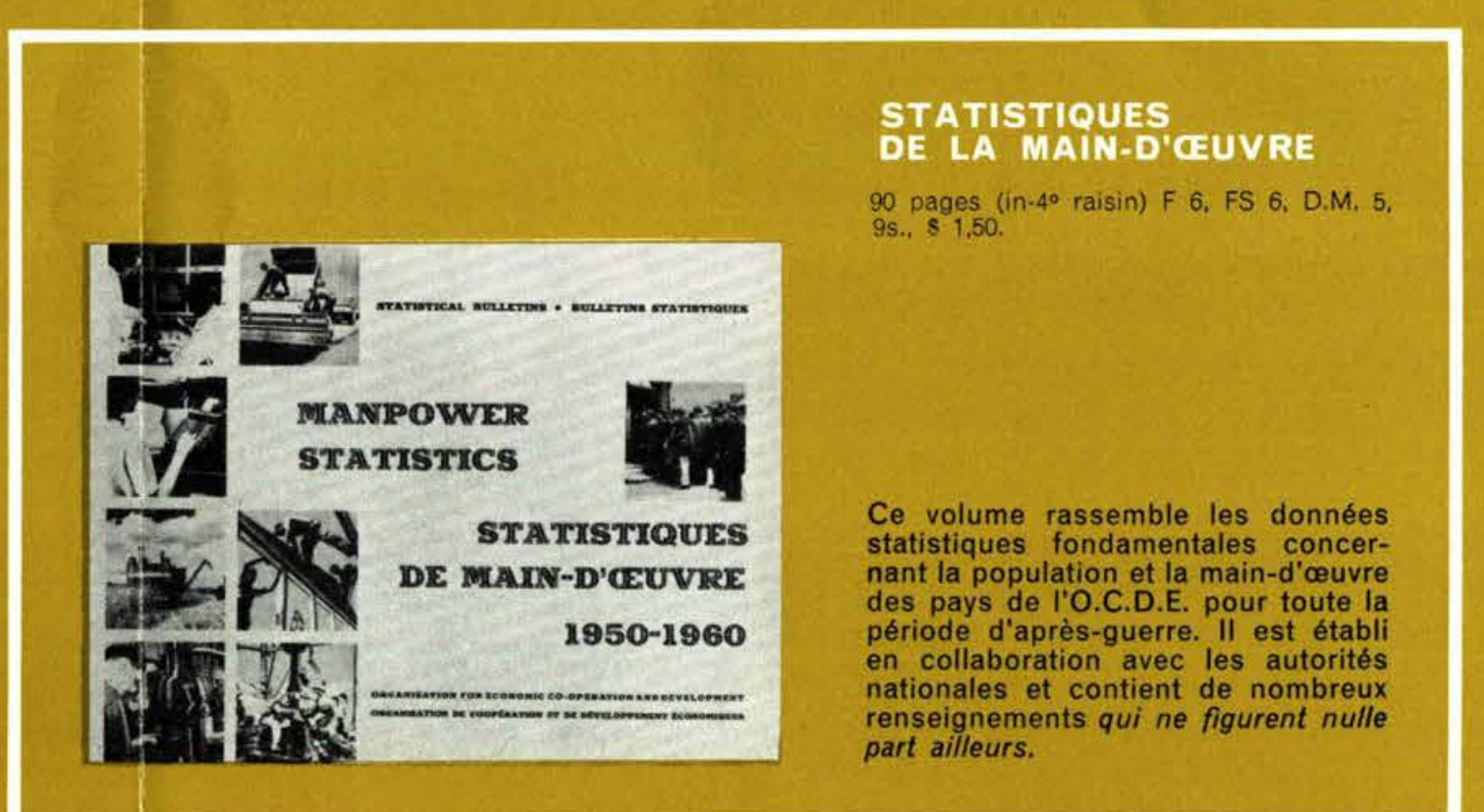

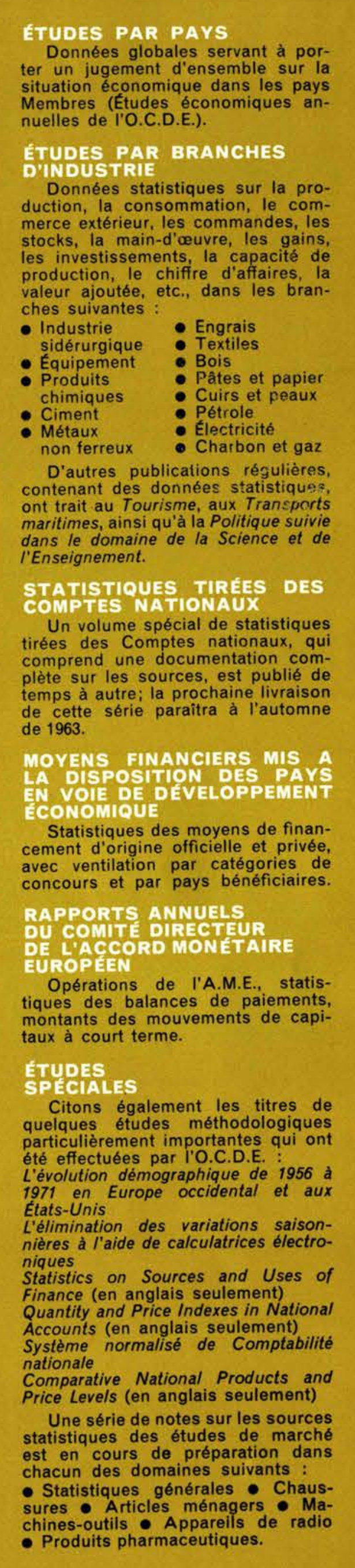




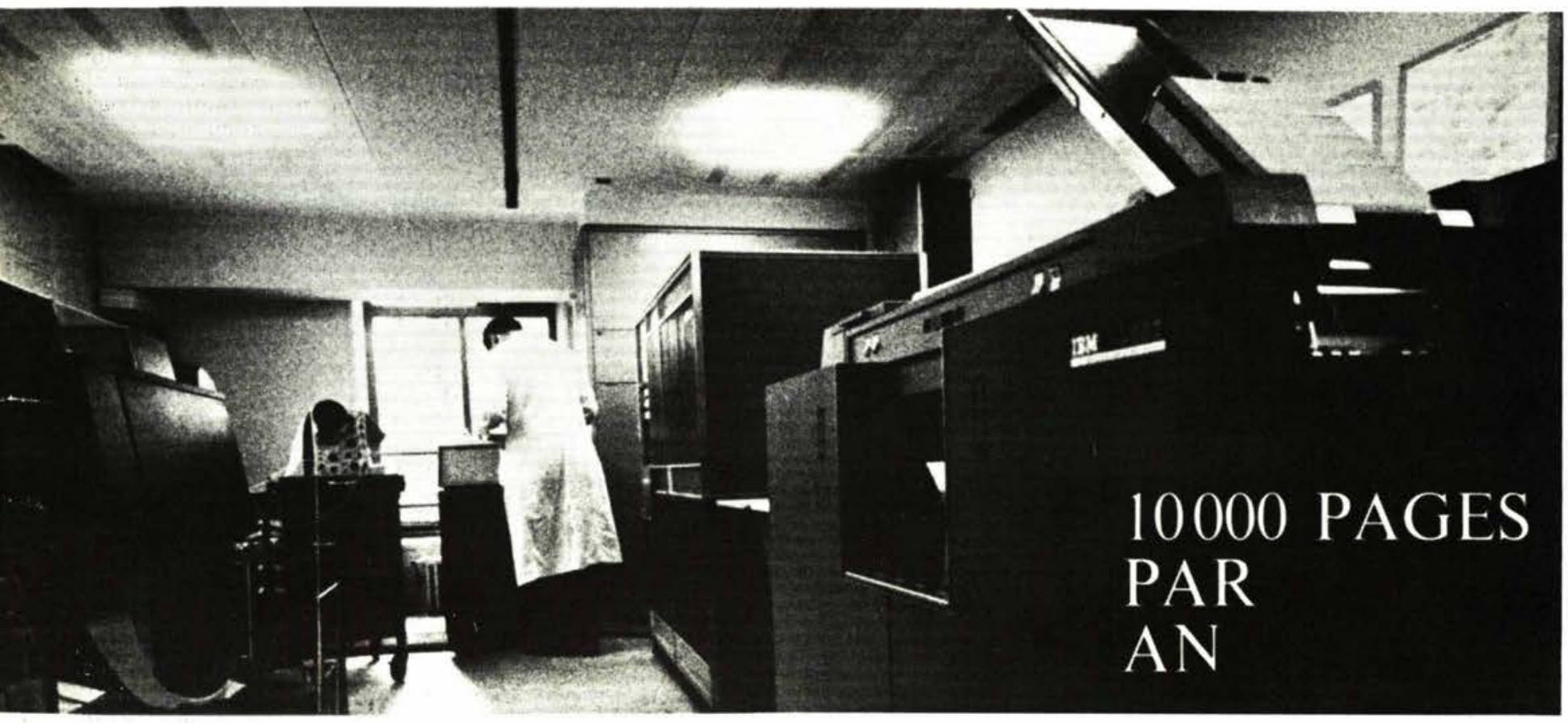

(suite de la page 19)

tout compte fait, un rôle des plus simples. Il consiste à réunir des renseignements provenant de sources extrêmement diverses, en donner la traduction dans un langage "commun" et à les présenter sous une forme condensée et facile à saisir. Par traduction, on n'entend pas seulement ici la traduction des termes techniques dans l'une ou l'autre des langues officielles de l'Organisation, encore que ceci soit important; il s'agit aussi de convertir des valeurs, des poids, des dimensions, etc., en unités communes, d'ajuster des indices de manière qu'ils aient la même année de référence, de regrouper les données élémentaires suivant une disposition normalisée, etc.

Cette tâche qui, à première vue, peut sembler fastidieuse et monotone, revêt en réalité une très grande importance. Si les gouvernements, les parlementaires, les organisations professionnelles, les syndicats, les instituts de recherche et les chefs d'entreprises devaient, chacun de leur côté, tirer les données chiffrées qui les intéressent d'une multitude de sources, il en résulterait un immense gaspillage d'efforts; de plus, on peut se demander si une pareille situation n'aurait pas nui à la coopération internationale étroite et étendue que nous connaissons aujourd'hui - à tel point qu'on est fondé à se demander si elle aurait été même concevable.

Une autre tâche de l'O.C.D.E. est d'ajuster les chiffres nationaux, de manière à les rendre aussi comparables que possible, par l'élimination des différences dans la définition des concepts sur lesquels ils reposent et dans le champ qu'ils recouvrent.

Parfois, cette tâche ne présente pas de difficultés. Certains pays, par exemple, font intervenir la construction dans le calcul de l'indice de leur production industrielle; d'autres ne le font pas. Un ajustement assez simple permet d'aligner ces indices sur la définition internationale normalisée, qui ne comprend pas la construction. Dans d'autres cas, il faut demander des renseignements supplémentaires ou des tableaux spéciaux aux services nationaux. Il arrive cependant que l'on ne puisse pas reconstituer des données comparables : c'est ainsi que la méthode utilisée pour réunir les statistiques du chômage aux États-Unis et au Canada diffère totalement de celle qu'emploient la plupart des pays européens. Quelquefois, il est possible d'estimer les chiffres que l'on obtiendrait à partir d'une base comparable; dans ce cas, il est précisé que l'on a affaire à des estimations. Lorsqu'on n'a pas recours à cette procédure ou lorsqu'on ne peut pas l'employer, il importe de bien mettre en garde l'utilisateur contre le danger que présenteraient des comparaisons viciées à la base.

L'Organisation a aussi pour rôle plus général et plus positif de collaborer avec les autorités des pays et avec les autres organisations internationales, en vue de chercher à améliorer sans cesse la portée et le champ des statistiques économiques nationales et internationales. Sur ce plan, les progrès ont été nécessairement graduels et n'ont rien eu de spectaculaire, car pendant longtemps les pays ont développé leurs services statistiques en fonction essentiellement de leurs objectifs nationaux et de leur situation propre.

Il faut cependant reconnaître que depuis la dernière guerre, la constitution d'un système de statistiques économiques comparables d'un pays à l'autre a fait d'immense progrès, dont le rythme s'est accéléré à mesure que l'interdépendance des pays Membres de l'Organisation s'affirmait.

L'apport de l'O.C.D.E. dans ce domaine revêt de multiples formes. Sa contribution la plus importante est sans doute la mise au point en collaboration avec d'autres organisations internationales, de définitions, de classifications et de méthodes internationales normalisées, qui sont sans cesse révisées à la lumière de l'expérience acquise et dont la formulation s'accompagne de conseils et de mises en garde à l'adresse des utilisateurs.

L'Organisation participe encore efficacement à cet effort par les confrontations de caractère officiel ou officieux qu'elle organise. Si un pays donné réussit à établir en cinq semaines des statistiques détaillées de son commerce extérieur, pourquoi un autre pays demande-t-il cing mois pour le faire? Ce genre de question permet souvent d'obtenir des améliorations dans un délai étonnamment bref.

L'O.C.D.E. s'efforce aussi de prêcher par l'exemple en adoptant des techniques statistiques modernes. Il n'est pas jusqu'à une certaine rivalité amicale entre les organisations internationales elles-mêmes qui ne puisse être profitable. 


\section{L'utilisation pratique des chiffres}

On est vite tenté de conclure que la quantité de données statistiques dont il serait souhaitable de disposer, est infinie. Ainsi, l'O.C.D.E. et sa devancière, l'O.E.C.E., ont toujours eu pour principe d'axer leur effort en matière de statistiques sur les domaines directement liés aux grandes questions de politique en cours d'examen.

Les Comptes nationaux, qui fournissent sous une forme quantitative une image complète et cohérente de l'économie d'un pays, avaient montré leur utilité à l'occasion du Programme de Relèvement Européen, et leur intérêt s'était révélé dépasser de loin les besoins du moment. A partir du cadre comptable utilisé à l'origine, un Système normalisé de Comptabilité nationale a été élaboré en 1952. en consultation avec les services statistiques nationaux et les organisations internationales. Ce Système a été maintes fois révisé par la suite, afin qu'il puisse servir à tout moment à l'étude des problèmes économiques très divers que les pays Membres, et par conséquent l'Organisation, pensent avoir à résoudre.

Les travaux de comptabilité nationale de l'Organisation ont eu pour corollaire logique l'évaluation de la puissance économique relative des pays et l'étude du pouvoir d'achat intérieur de leurs monnaies. La première étude comparative de l'O.C.D.E. sur les produits nationaux et les niveaux de prix a été publiée en 1954. Elle fut suivie en 1958 d'une nouvelle étude qui a porté sur un plus grand nombre de pays et mis à jour les résultats antérieurs. Les travaux se poursuivent dans ce domaine. Les deux exemples suivants montrent à quel genre de constatations ces recherches statistiques peuvent aboutir. En 1955, la valeur de la production moyenne par habitant en Europe s'établissait à environ 750 dollars, lorsqu'on la convertissait aux taux de change officiels; par contre, si on la calculait d'après les prix relatifs des produits correspondants aux États-Unis, on arrivait à une évaluation bien supérieure (1 110 dollars). C'est dire que lorsque l'on compare des niveaux de production en se servant des taux de change officiels, le résultat peut différer considérablement, en plus ou en moins, de celui que donnerait une méthode de mesure manifestement mieux adaptée. Ces études ont permís de chiffrer pour la première fois les distorsions qui peuvent se produire de ce fait. On a constaté aussi qu'en 1955 un billet d'un dollar, converti en sterling au taux de change officiel et dépensé au Royaume-Uni conformément à la manière dont le consommateur britannique répartissait alors ses achats, permettait d'acquérir près de $50 \%$ de produits de plus que s’il avait été consacré à l'achat des mêmes biens aux États-Unis.

L'O.C.D.E. a pris la succession de l'O.E.C.E. en 1961, avec un mandat élargi, et les travaux statistiques de la nouvelle Organisation ont reflété l'extension de son champ d'activité et de ses objectifs. Un exemple le montrera. Reconnaissant les liens étroits qui existent entre la science, l'enseignement et le taux de croissance économique, l'O.C.D.E. examine régulièrement la question du potentiel scientifique et technique des pays Membres, en confrontant leurs besoins et leurs ressources. Les données que cet examen requiert, vont du montant des gains que perçoit le personnel technique hautement qualifié, au pourcentage de la dépense nationale que la recherche absorbe.
Il y a quelques années, on ne disposait dans ce domaine que d'une documentation fragmentaire, dépourvue de tout caractère systématique et qui ne permettait pas de faire des comparaisons. Bien qu'encore aujourd'hui l'on ne dispose pas de toutes les données statistiques voulues, de grands progrès ont été accomplis, et l'O.C.D.E. publie régulièrement des études par pays sur la politique suivie dans le domaine des sciences et de l'enseignement; ces rapports sont fondés dans une très large mesure sur des chiffres qui n'auraient jamais pu être réunis si l'Organisation n'avait pas activement encouragé et coordonné les efforts des pays.

De même, en raison des objectifs que l'O.C.D.E. poursuit dans le domaine de l'aide, il a fallu réunir des données sur les activités que les pays Membre déploient en vue d'obtenir que les pays sous-développés utilisent au mieux les ressources économiques mises à leur disposition. Toute une documentation est recueillie et publiée sur les moyens financiers fournis aux pays en voie de développement économique; on y trouve le détail des financements officiels et privés, ventilés par catégories de concours et par pays bénéficiaires. L'Organisation calcule le pourcentage que l'aide représente dans le Produit national brut des pays donateurs, et elle évalue les conséquences que cette aide peut avoir sur leur balance des paiements, ce qui permet de mettre en parallèle les contributions de ces pays avec les moyens financiers qu'ils seraient en mesure de fournir.

Enfin, et c'est peut-être là son activité la plus importante, l'Organisation ne peut songer atteindre efficacement ses objectifs dans le domaine de la coordination des politiques économiques suivies par les pays Membres sur le plan national et international, que si elle dispose d'une évaluation précise et à jour de la situation économique dans les pays du monde occidental. A cet effet, il faut en permanence passer au crible les renseignements reçus et les analyser à l'aide des techniques modernes les plus puissantes.

Un bon exemple des travaux que l'Organisation exécute dans ce domaine, est l'utilisation de données "désaisonnalisées". Depuis longtemps, on savait que la plupart des phénomènes qui font l'objet de mesures statistiques sont soumis à des variations saisonnières (dans le cas du chômage, nettement plus de la moitié de la variation moyenne que la plupart des séries font apparaître d'un mois à l'autre, a un caractère saisonnier); pour évaluer de façon précise la tendance profonde de ces phénomènes, il faut done isoler la composante saisonnière. Des méthodes de correction des variations saisonnières reposant sur l'emploi de calculatrices électroniques étaient étudiées aux États-Unis depuis 1955. L'Organisation a participé à leur mise au point. Au cours d'une réunion qui s'est tenue en 1960, les progrès accomplis ont été examinés et des amćliorations nouvelles ont été proposées. L'intérêt que présentent des données "désaisonnalisées " étant maintenant plus largement reconnu, ces méthodes sont appliquées à un ensemble de plus en plus grand de séries statistiques par des pays toujours plus nombreux. L'O.C.D.E. elle-même publie plus de 250 séries corrigées des variations saisonnières, et le nombre des séries traitées sera prochainement augmenté grâce à un programme qui a été spécialement écrit pour la calculatrice électronique de l'Organisation. 


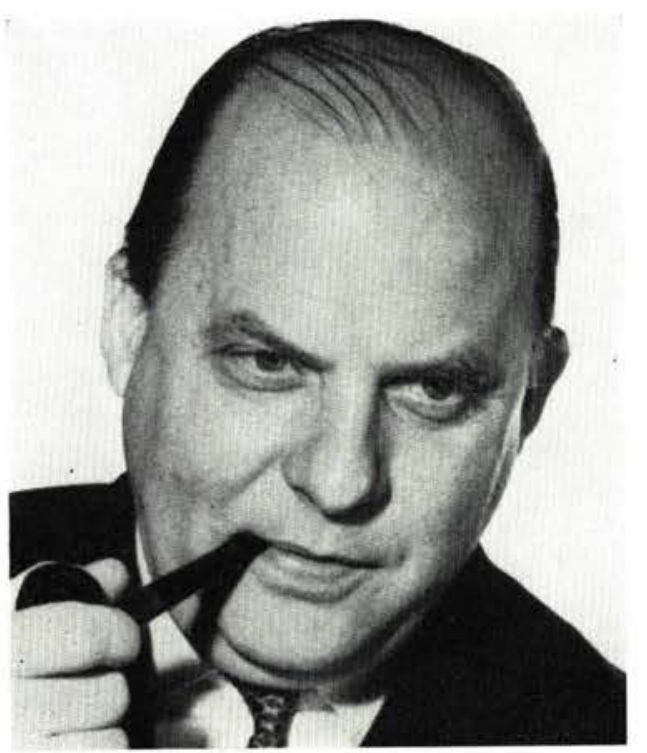

par

HENNING FRIIS

Président du Comité

du Personnel Scientifique et Technique

\section{COMMENT \\ EVITER UN}

RALENTISSEMENT

DU PROGRES

ECONOMIQUE

\section{l'action de l'OCDE}

\author{
pour parer au manque de personnel \\ scientifique et technique
}

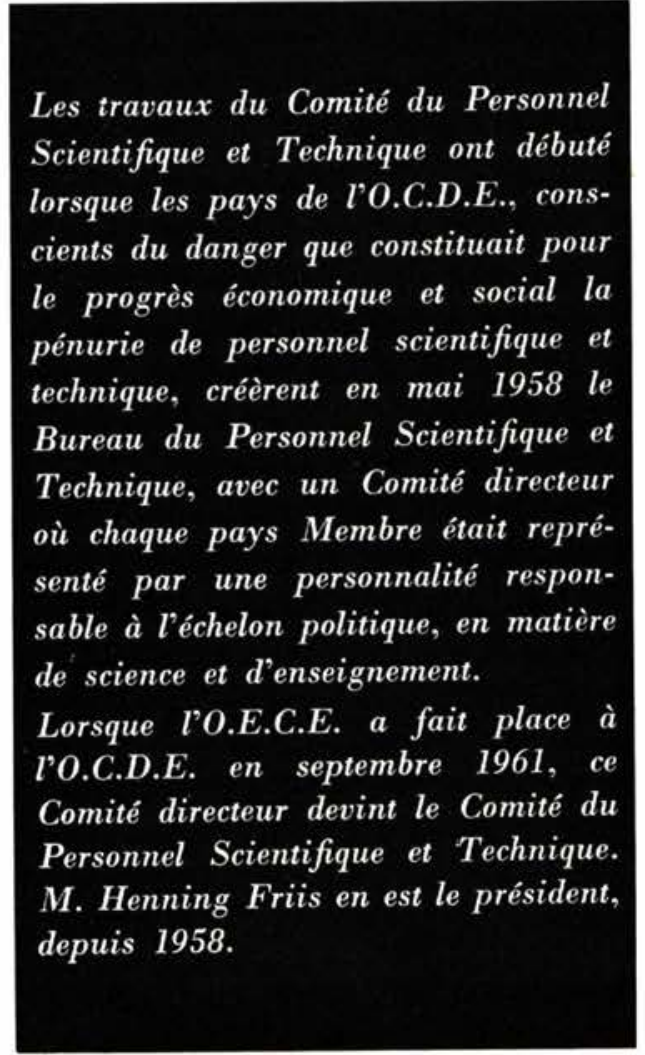

D ans notre monde moderne, Fexpansion économique dépend de plus en plus de la disponibilité de personnel scientifique, technique et hautement qualifié, c'est-à-dire du développement de l'enseignement qui forme ce personnel. Les politiques d'accélération de la croissance économique auront vraisemblablement pour effet d'accentuer encore, au cours des années 1960-70, cette dépendance, car de telles politiques impliquent, entre autres choses, un taux plus élevé d'investissement en capital, et d'innovation technique, ce qui nécessite beaucoup de personnel qualifié.

En outre, un rythme de progrès plus rapide implique également une plus grande capacité d'organisation d'un monde en transformation technique et sociale - nouveaux modes de production, nouvelles écoles, nouveaux services sociaux, etc. - et pour tout cela, il faut aussi un personnel ayant reçu une éducation et une formation d'un niveau élevé.

En fait, à l'époque où le Comité du Personnel Scientifique et Technique élaborait son programme, le développement économique de l'Europe d'après-guerre était déjà entré dans une phase nouvelle : la "reconstruction " était achevée et l'on se préoccupait surtout d'expansion équilibrée. Ce programme a été inspiré par la conviction qu'un surcroit d'investissements consacrés à la formation du personnel scientifique et technique - et à l'enseignement général, base de cette formation est désormais un élément essentiel de toute politique économique progressiste.

Dans sa politique et dans les opérations qu'il entreprend, le Comité du Personnel Scientifique et Technique a pleinement reconnu ce lien entre expansion de l'enseignement et possibilités de progrès économique futur. Un pas décisif vers la définition d'une politique qui rattache les besoins de l'enseignement aux besoins économiques a été accompli lors d'une importante réunion, qui s'est tenue à l'échelon politique à 


\section{COMMENT EVITER UN RALENTISSEMENT DU PROGRES ECONOMIQUE}

Washington en octobre 1961. Des objectifs d'investissements dans l'enseignement jusqu'en $1970 \mathrm{y}$ furent discutés, et la nécessité y fut généralement admise de mettre en cuvre pour la zone de l'O.C.D.E., un plan à long terme d'investissements dans l'enseignement.

Une fois cet objectif posé, il a fallu inciter les théoriciens à réétudier d'une façon nouvelle les questions complexes, de principe, de méthode et de politique, qui se posent lorsqu'on veut avoir une vue d'ensemble du processus par lequel l'enseignement contribue au développement du système économique. C'est à cette intention qu'a été créé le Groupe d'études sur les aspects économiques de l'enseignement, où se trouvent réunis des experts en science économique indépendants et des personnalités responsables au niveau politique, qui peuvent en toute liberté se consacrer à l'étude des problèmes théoriques et pratiques en question. Le Groupe d'études s'occupe actuellement d'évaluer, dans un nombre important de pays Membres, les rapports existants entre investissement dans l'enseignement et croissance économique. Il organisera prochainement une conférence où d'éminents économistes des pays de l'O.C.D.E., examineront les données concrètes dont on dispose pour évaluer à quel point l'enseignement et la recherche scientifique peuvent expliquer la croissance économique. Le Groupe d'études examinera également comment l'adaptation de l'enseignement aux besoins de l'économie réagit sur la structure et l'organisation des systèmes d'enseignement.

L'Organisation ne s'est pas contentée de susciter de nouveaux travaux théoriques; elle a également encouragé la création dans les pays Membres de l'O.C.D.E. de mécanismes de planification gràce auxquels les objectifs d'expansion de l'enseignement et les politiques qui permettront d'atteindre ces objectifs, pourront ètre formulés et poursuivis dans le cadre de la politique générale du pays. Le Comité collabore avec les pays Membres grâce à deux programmes majeurs qui intéressent l'un la région méditerranéenne et l'autre les pays d'Europe septentrionale.

Le Programme Régional Méditerranéen, qui a été lancé en 1960 à l'instigation du Comité (et avec la participation active de l'O.C.D.E. pour l'organisation, le financement et le recrutement du personnel nécessaire au projet), a conduit les Gouvernements d'Espagne, de Grèce, d'Italie, du Portugal, de Turquie et de Yougoslavie à constituer des équipes nationales chargées de formuler des propositions en vue de la planification intégrée de l'enseignement et du développement économique. La tàche de ces équipes consiste à étudier dans quelle mesure, et comment, les moyens d'enseignement devront ètre développés au cours des quinze prochaines années pour que les objectifs d'expansion économique à long terme puissent ètre atteints. Il s'agit d'une étude d'ensemble qui tient compte des nécessités économiques. Les six équipes nationales doivent déposer leur rapport dans le courant de cette année.

Bien que les besoins et les problèmes d'enseignement dans la région méditerranéenne soient tout à fait différents de ceux des pays plus industrialisés, il est d'ores et déjà manifeste que les méthodes mises au point par les équipes du Projet Régional Méditerranéen sont susceptibles d'une application générale. Aussi le Comité a-t-il lancé un Programme de prévisions et d'investissements dans l'enseignement auquel participent maintenant plusieurs pays d'Europe septentrionale.

Il nous a été particulièrement agréable, à mes collègues du Comité et à moi-mème, de constater que les idées nouvelles dont nous nous étions faits les promoteurs obtenaient une faveur rapidement croissante auprès des Ministres. Les travaux du Comité sur l'investissement dans l'enseignement ont été récemment approuvés par les Ministres européens de l'Éducation dont une Résolution, officiellement adoptée lors de leur $3^{\mathrm{e}}$ conférence, à Rome en 1962, exprime le vœu " que les travaux entrepris dans ce domaine par l'O.C.D.E. soient activement poussés, que la coopération entre nations européennes dans la poursuite de ces objectifs soit facilitée par les rapprochements et les comparaisons que suscite cette Organisation et qu'en liaison avec les autres organisations intéressées, notamment le Conseil de l'Europe, elle informe les Conférences subséquentes des Ministres européens de l'Éducation des difficultés rencontrées et des résultats obtenus ".

\section{CONFRONTATION DES POLITIQUES NATIONALES}

Orientés selon de nouvelles conceptions, ces travaux sur l'investissement dans l'enseignement et la transformation de l'enseignement scientifique et technique rendue nécessaire pour répondre aux exigences de l'expansion économique future, ont pour principal objectif d'aider chacun des Gouvernements Membres à développer et à préciser sa politique. L'O.E.C.E. et aujourd'hui l'O.C.D.E. offre à ses Membres, et c'est là une réussite unique en son genre, un terrain de rencontre qui leur permet d'avoir des discussions franches et constructives sur leurs problèmes en matière de politique. Le Comité du Personnel Scientifique et Technique perpétue cette tradition de l'Organisation et son "Programme d'examen par pays " est à la base de ses travaux. Le but du programme d'examen par pays est d'examiner les problèmes que rencontrent les pays Membres en matière de personnel scientifique et technique, de politique et de planification concernant l'enseignement, et de soumettre ces problèmes à une étude critique mais constructive. A l'issue de ces examens, le Comité ne formule pas de conclusions précises, mais l'étude et la discussion elles-mèmes constituent une importante contribution au débat national sur des questions essentielles, et peuvent ainsi apporter une aide aux autorités nationales aux prises avec ces problèmes.

La méthode adoptée pour ces examens consiste à inviter deux ou trois personnalités éminentes dans le domaine de la science et de l'éducation, à procéder, en qualité "d'examinateurs", à une enquête sur place. Leur rapport sert ensuite au Comité de document de base lors d'une séance dite de "confronta- 


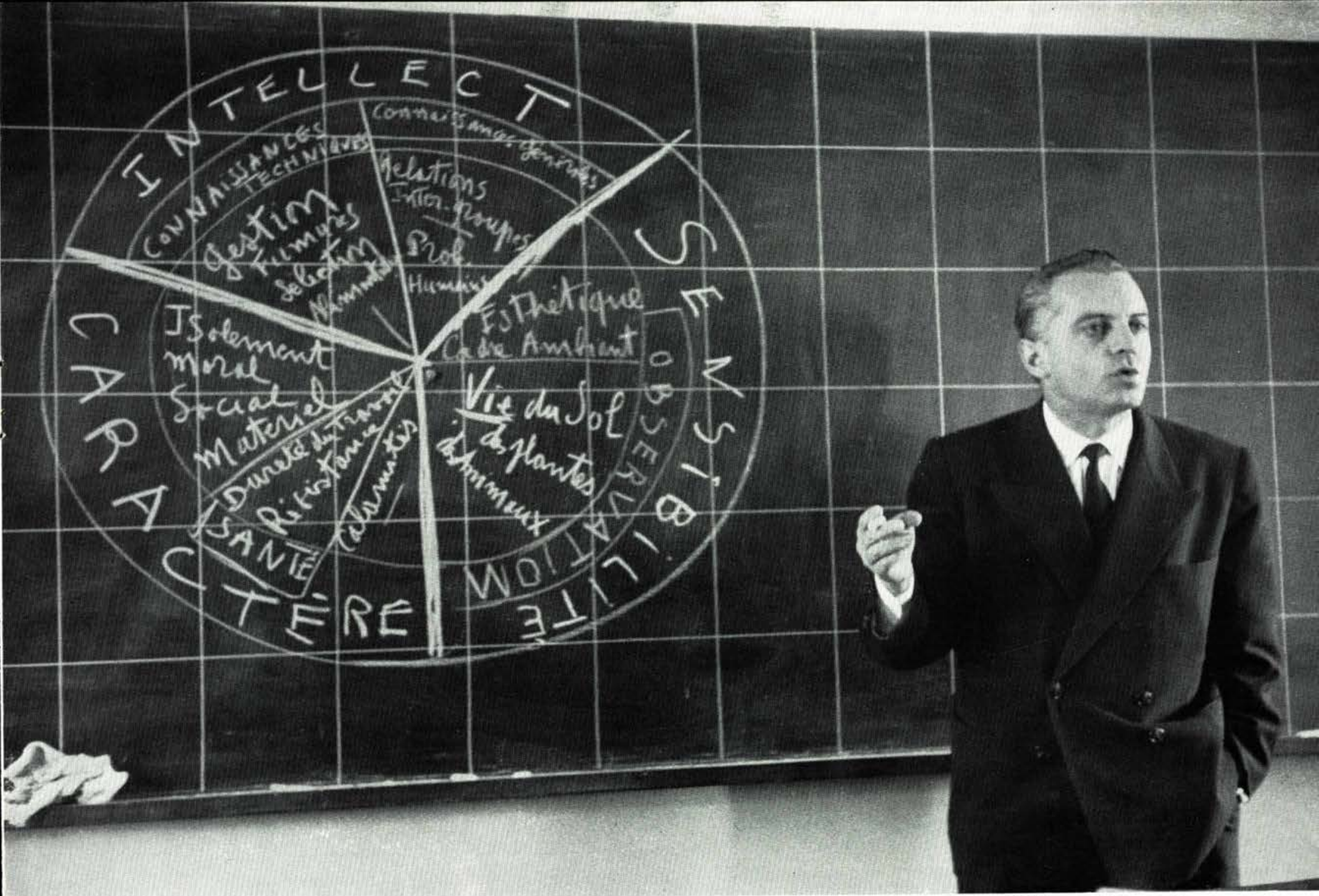

M. Bretones, consultant de l'O.C.D.E., donne un cours à l'Institut des Hautes Études Agronomiques Méditerranéennes de Montpellier (France).

tion " pendant laquelle les représentants du pays intéressé répondent aux questions que leur posent les examinateurs et les membres du Comité. Le Rapport final d'examen, publié par l'O.C.D.E., recoit une large diffusion, non seulement dans le pays " examiné " mais dans les autres pays Membres et non-membres, qui ont à faire face à des problèmes analogues.

Les examens par pays fournissent également au Comité une bonne base pour la préparation de ses programmes d'opération. On ne saurait décrire ici chacun des quelque 20 projets auxquels le Comité se consacrera pendant l'année. Nous nous contenterons donc de deux ou trois exemples qui suffiront à donner une idée des travaux du Comité.

\section{RÉVOLUTION DANS L'ENSEIGNEMENT DES SCIENCES ET DES TECHNIQUES}

Le Comité a posé comme principe fondamental de sa politique qu'on ne peut traiter correctement le problème du potentiel scientifique des pays Membres que dans une perspective à long terme. En dernière analyse, ceci n'est possible qu'au prix d'une expansion et d'un perfectionnement fondamental de l'enseignement général, plus particulièrement d'une réforme de l'enseignement des sciences et des mathéma- tiques dès le niveau des études secondaires, pour doter la génération montante de l'outil intellectuel qui lui permettra d'assimiler et de faire progresser à son tour les sciences et les techniques modernes, et au sens le plus général, de comprendre le monde contemporain.

Aussi l'Organisation a-t-elle fait un effort considérable pour encourager les pays Membres à réviser leurs méthodes d'enseignement des sciences. Un nouveau programme d'enseignement des mathématiques a été proposé et a trouvé une large audience; une tentative est actuellement en cours pour moderniser de facon fondamentale l'enseignement de la chimie en faisant appel aux connaissances actuelles sur la nature des liaisons chimiques; les méthodes d'enseignement expérimentales et théoriques de la physique, qui sont en cours de mise au point aux États-Unis et dans d'autres pays, ont été très attentivement considérées et actuellement on travaille à réorganiser dans le mème esprit l'enseignement de la biologie. On ne s'est pas contenté de ces études générales sur les nouvelles façons d'enseigner les sciences et les mathématiques; des colloques régionaux ont été organisés pour examiner comment introduire les idées nouvelles dans les divers systèmes d'enseignement et l'on s'est efforcé d'encourager l'application de ces nouvelles méthodes à titre expérimental dans des classes-pilotes. Il y a là pour l'O.C.D.E. un effort majeur et de longue haleine qui va pro- 


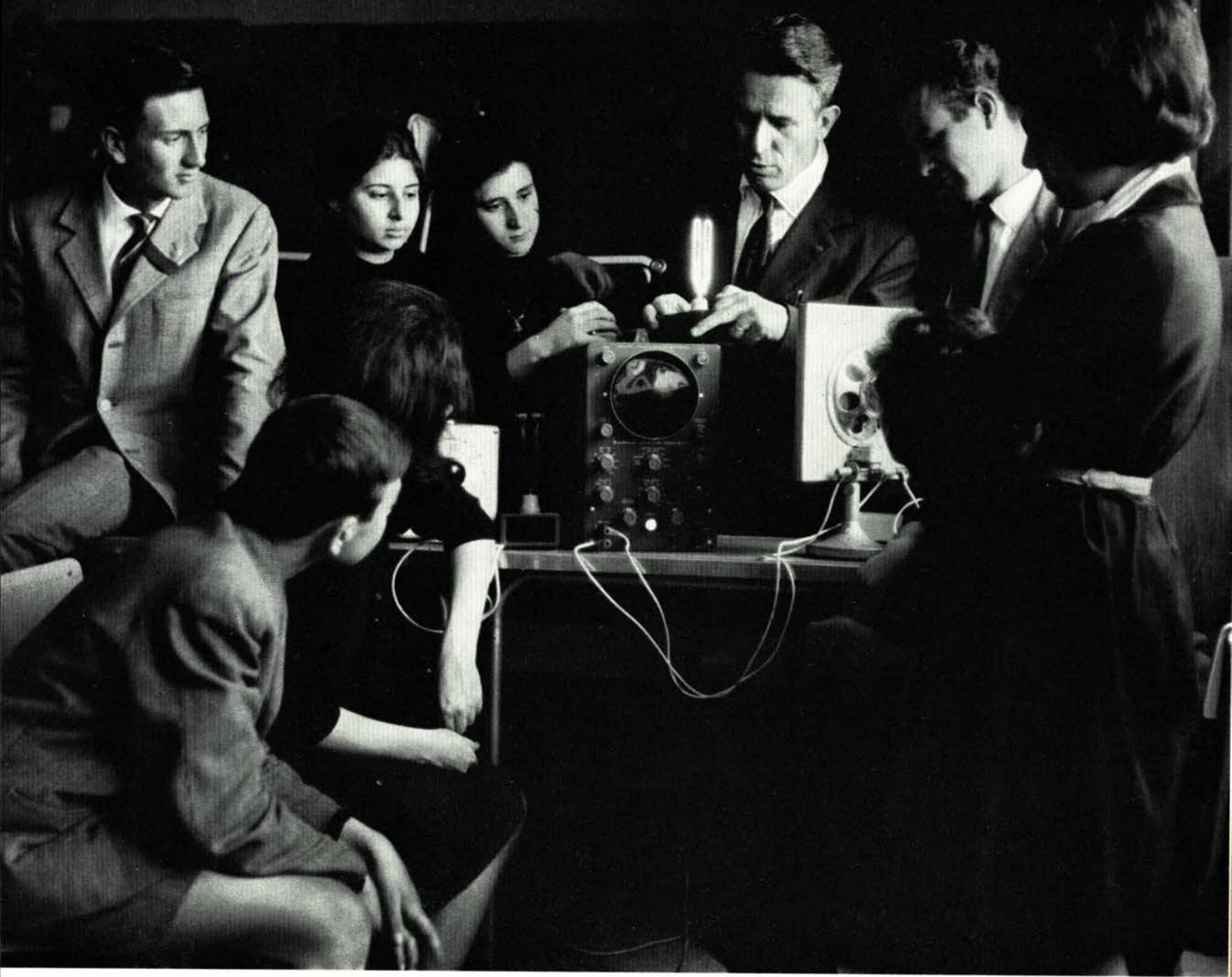

Un professeur voyageant avec l'Unité Mobile de l'O.C.D.E. en Sicile explique le fonctionnement d'un oscillographe à des élèves de Raguse (Sicile).

gressivement s'étendre et gagner en profondeur.

Il est indispensable, en particulier, de faire appel aux techniques modernes telles que la télévision et le film, de façon à mettre sous les yeux des élèves dans la classe mème une image de la science qu'aucun autre moyen ne pourrait leur fournir facilement.

\section{« LES POINTS DE CROISSANCE » DANS LA RECHERCHE \\ ET L'ENSEIGNEMENT SCIENTIFIQUE DE TYPE AVANCÉ}

Le Système des points de croissance de l'O.C.D.E. imaginé par le Comité du Personnel Scientifique et Technique, repose sur l'idée suivante: il devient de plus en plus manifeste qu'en raison de l'extension et du coùt croissant de la recherche, les institutions scientifiques et mème les pays vont éprouver des difficultés à poursuivre seuls, à une échelle suffisante pour ètre efficace, leur effort scientifique dans les domaines spécialisés de type avancé; il faudrait donc encourager certaines institutions bien choisies à assumer un rỏle scientifique à l'échelon régional voire européen. Ce système reprend en somme, pour l'amplifier et accélérer ses effets par l'action internationale, le processus traditionnel qui permettait aux professeurs éminents de s'attirer les étudiants les plus brillants, sans considération de nationalité. Ce sont les pays scandinaves qui ont jusqu'ici développé ce système le plus activement; les "points de croissance " qu'ils ont créés avec l'appui de l'O.C.D.E. sont spécialisés dans l'acoustique, la corrosion des métaux, le génie chimique, l'automation, les techniques biologiques, la spectographie de masse des composés organiques, la transmission de la parole, et la calorimétrie chimique. On envisage actuellement l'extention du système et son adaptation à certains autres pays Membres et en particulier à ceux en voie de développement.

Au moment où dans le monde entier les Gouvernements cherchent les moyens de développer à l'échelon régional les facilités de recherche et de formation scientifique de type avancé devenues 


\section{COMMENT EVITER UN RALENTISSEMENT DU PROGRES ECONOMIQUE}

trop coùteuses en hommes et en argent à l'échelle de la nation, le système des "points de croissance" de l'O.C.D.E. mérite de retenir l'attention. Son principe essentiel réside en effet dans l'idée de renforcer et d'internationaliser les institutions nationales existantes - au lieu de créer de nouveaux organismes internationaux, coùteux et entraînant parfois un gaspillage d'efforts.

\section{LA RÉGION MÉDITERRANÉENNE ZONE PILOTE DU DÉVELOPPEMENT}

Le Comité a accordé une attention spéciale aux besoins des pays Membres de la région méditerranéenne auxquels le développement scientifique et celui de l'enseignement posent des problèmes spéciaux. Le Comité a la conviction que, dans le passé on n'a pas attaché suffisamment d'importance aux investissements consacrés à la science et à l'enseignement dans les programmes de développement économique et dans les programmes d'assistance technique. On n'a pas su reconnaitre le rỏle vital du développement des ressources humaines, et en particulier d'une main-d'œuvre hautement qualifiée, dans la croissance économique. Faute d'avoir su développer leur capital humain, bien des pays développés et sous-développés se trouvent limités dans leurs possibilités de mise en valeur de nouveaux capitaux physiques. En outre, ce déséquilibre entre l'expansion du capital humain et du capital physique est d'autant plus grave que les investissements consacrés à l'enseignement et à la formation professionnelle sont caractérisés par une maturation particulièrement lente et il faut attendre longtemps avant d'en obtenir des résultats sous forme de personnel qualifié.

C'est ce qui fait que le Comité attache une grande importance à l'évaluation des besoins à long terme en matière de développement de l'enseignement dans la région méditerranéenne (grâce au Projet Régional Méditerranéen mentionné ci-dessus) et à l'élaboration de politiques qui permettent de combler l'écart entre les besoins et les ressources disponibles pour le développement de l'enseignement. Des projets pilotes, qui permettront d'explorer certaines laçons de combler cet écart, ont été lancés en Espagne, en Grèce, en Italie, au Portugal, en Turquie et en Yougoslavie ; ils ont pour objectif, entre autres, la création de centres nationaux de production de matériel scolaire pour l'enseignement des sciences, le renforcement des facilités scientifiques dans certaines universités, l'expérimentation de certaines réformes de programmes d'études et la mise en service d'" unités mobiles " pour l'enseignement des sciences.

La hardiesse que les Gouvernements des pays méditerranéens apportent dans l'expérimentation de ces nouvelles méthodes de planification de l'enseignement n'est pas seulement un puissant stimulant pour leur propre développement national, elle suscite aussi de nouvelles idées et de nouvelles méthodes qui peuvent intéresser tous les pays Membres de l'O.C.D.E. C'est pourquoi le Comité a organisé un programme de cours de formation qui permettra à de jeunes spécialistes d'Europe septentrionale et
d'Amérique du Nord d'assimiler les résultats de cette expérience et de les transposer dans leur propre pays et dans les pays sous-développés; le succès remporté par ce projet a démontré le grand intérèt que portent les jeunes économistes et statisticiens des pays Membres de l'O.C.D.E. à se préparer à traiter efficacement les problèmes des pays sous-développés.

La planification à l'échelle de la nation, surtout lorsqu'on veut l'appliquer à une entité touchant aussi directement la personne humaine que l'enseignement, suscite encore dans bien des milieux une certaine suspicion. Et pourtant, il est difficile de ne pas voir que si l'on veut mettre le progrès incessant des sciences au service de la prospérité nationale et du mieux-être de chacun, il faudra qu'une proportion sans cesse croissante de la population accède à un niveau plus élevé d'éducation et de compétence technique. Le jour n’est plus très éloigné où ce ne seront plus seulement les savants, les ingénieurs et techniciens, mais la plupart des travailleurs de l'industrie, chaque citoyen et les politiciens euxmèmes qui devront avoir, des principes et des problèmes de la science, une compréhension plus assurée qu’il n’était jusqu'ici nécessaire. En outre, l'évolution sociale exerce une pression de plus en plus forte dans le sens de l'élargissement des possibilités d'éducation. L'éducation ne peut donc plus être considérée comme quelque chose de séparé du courant principal de la vie économique du pays. C'est parce que les Membres de l'O.C.D.E. ont reconnu que l'éducation prépare les voies où s'engage l'économie, que les problèmes d'enseignement occupent aujourd'hui une place si importante dans les activités de l'Organisation.

\section{UN DES EXEMPLES LES PLUS INTÉRESSANTS DE COLLABORATION INTERNATIONALE}

L'activité du Comité du Personnel Scientifique et Technique au cours des cinq dernières années offre un exemple intéressant et instructif de collaboration internationale. Les exemples cités cidessus montrent qu'il a réussi non seulement à élaborer de nouvelles conceptions et de nouvelles méthodes en matière de politique, mais aussi à lancer un nombre considérable d'activité pratiques et tout à fait concrètes, se traduisant par la collaboration de divers groupes de spécialistes des pays Membres. C'est peut-être cette fusion effective des activités à l'échelon "politique" et à l'échelon "opérationnel " qui constitue l'une des réalisations les plus significatives du Comité. Si l'importance accordée par le Comité à la réflexion politique a permis d'orienter constamment les actions internationales vers les tàches prioritaires - ce qui est évidemment essentiel pour une organisation internationale - les programmes d'opération ont fourni par contre ces multiples contacts avec les réalités qui ont permis à l'O.C.D.E. de sortir de l'isolement du Château de la Muette pour se plonger dans les problèmes pratiques de ses pays Membres. 
Les baleines de corset, exportées à destination de l'un des grands pays de l'O.C.D.E., doivent porter individuellement l'indication du pays d'origine bien que ces baleines ne soient jamais visibles une fois qu'elles sont incorporées dans le produit fabriqué. De même au dos des plaques d'identité pour colliers de chiens, doit figurer le pays d'origine. Et pourtant, qui peut savoir où elles ont été fabriqués lorsqu'elles sont montées sur des colliers.

Des règlements de ce genre, même s'ils n'ont qu'une importance mineure, entravent les échanges puisque l'exportateur doit satisfaire à des conditions différentes selon le marché national auquel ses produits sont destinés. Les règles de marquage ne sont toutefois qu'un exemple, et probablement l'un des moins importants, dans toute une série de règlements administratifs et techniques qui influencent à des degrés divers le mouvement du commerce international.

Dans le passé, les droits de douane et les restrictions quantitatives imposées aux importations constituaient les barrières normales aux échanges. Progressivement les restrictions quantitatives ont été substantiellement réduites et, en fait, virtuellement éliminées pour les importations de produits non agricoles; les tarifs douaniers ont été abaissés de manière importante et l'on s'efforce constamment de faire de nouveaux progrès dans ce domaine.

A mesure que ces barrières disparaissent, les effets restrictifs des règlements administratifs et techniques en vigueur deviennent plus apparents; il devient aussi plus tentant d'utiliser certains de ces règlements pour remplacer les modes normaux de protection.

$\mathbf{L}$ ses règlements administratifs et techniques susceptibles d'entraver les échanges constituent, en fait, deux groupes distincts. Dans un premier groupe figurent les règlements qui sont en relation directe avec les échanges : c'est le cas par exemple des formalités douanières, des règles concernant la classification et l'évaluation des produits en vue de l'application des tarifs douaniers ainsi que des procédures de délivrance des licences, des permis et des certificats d'importation. Un certain nombre de ces règlements ont été mis en vigueur entre les deux guerres, d'autres pendant la période difficile qui a suivi immédiatement la dernière guerre alors que les difficultés de la balance des paiements obligeaient des pays même profondément attachés à une politique libérale des échanges à recourir au contrôle des changes et

\section{les règlements administratifis} et techniques opposent aux échanges des obstacles peu connus

aux licences d'importation. Les monnaies étant de nouveau convertibles et les restrictions quantitatives ayant pu être assouplies, le mécanisme autrefois nécessaire pour contrôler les importations est devenu dans une large mesure superflu. Cependant, des contrôles de ce genre sont rarement supprimés aussitôt qu'il serait possible. Ils sont maintenus soit simplement à des fins statistiques, soit parce que l'on craint qu'une fois supprimés ils soient difficiles à rétablir. Ainsi, même lorsque les importations ne subissent plus aucune restriction, il peut subsister une procédure de délivrance de licences d'importation; le fait de maintenir un tel mécanisme, alors qu'il n'est plus nécessaire, peut offrir une certaine protection aux producteurs nationaux.

Même si elles ont un objet utile, les formalités relatives aux importations et aux exportations peuvent constituer un obstacle aux échanges en raison du temps qu'il faut pour les accomplir. De plus, la complexité de bien des formalités auxquelles doivent se conformer ceux qui souhaitent importer ou exporter des marchandises constitue un facteur nuisible que l'on ne peut considérer que comme un obstacle aux échanges.

Ainsi l'importateur peut être obligé de remplir plusieurs formulaires extrêmement détaillés, analogues mais non identiques les uns aux autres, et de fournir pour chacun un grand nombre de copies. Il peut devoir attendre des semaines ou même des mois avant de recevoir sa licence d'importation. En outre, les règles de classification et d'évaluation des produits peuvent être si complexes qu'il lui est impossible de savoir exactement, au moment où il passe une commande, quel sera le montant des droits d'importation qu'il devra acquitter; il est enfin obligé de consacrer du temps à se tenir au courant 
d'une foule de règles constamment variables ou de s'assurer les services d'une autre personne chargée de le faire.

Le second groupe de règles techniques et administratives comprend toutes sortes de règles n'ayant pas directement trait aux échanges mais qui concernent la sécurité, la santé publique, le contrôle de qualité, la prévention des fraudes ou la rationalisation de la production. Un pays peut, par exemple, exiger que les semences et le bétail importés soient examinés au point de vue de différentes maladies, que les machines agricoles répondent à certaines normes de sécurité, qu'avant d'être mis en vente, les engrais soient soumis à une analyse chimique et les produits pharmaceutiques à des essais de la part des autorités médicales. Dans la plupart des cas, il ne fait aucun doute que des règlements de cette sorte sont parfaitement justifiés, mais leur diversité d'un pays à l'autre, leur sévérité qui dépasse parfois ce qui est nécessaire, et souvent leur complexité, constituent un obstacle aux échanges et un élément de protection pour les producteurs nationaux.

L O.C.D.E. se préoccupe de plus en plus du problème si complexe des règlements administratifs et techniques. Elle a pour objectif d'éliminer les règlements inutiles ou discriminatoires, sans porter préjudice aux intérêts légitimes qui peuvent être en cause; de réduire les délais d'adaptation de la législation à l'évolution de la technologie en vue d'éliminer au maximum les dispositions réglementaires désuètes; enfin, de faciliter les échanges en encourageant la simplification des procédures et la normalisation des règles dans les différents pays. En 1962, le Conseil de l'Organisation a recommandé aux Gouvernements des pays Membres d'examiner périodiquement les règlements administratifs et techniques appliqués dans leurs pays, en vue de supprimer les dispositions qui ne sont pas indispensables et celles qui entravent les échanges; il a également institué une procédure permettant à l'O.C.D.E. de se saisir des problèmes qui pourraient se présenter dans ce domaine.

En raison de la diversité des règlements techniques et administratifs, l'Organisation a adopté pour atteindre ses objectifs, une méthode tout à fait souple. Dans certains cas, un pays dont les exportateurs ont éprouvé des difficultés en raison des règlements appliqués par un autre pays Membre peut soumettre le problème à l'O.C.D.E. qui s'efforcera de régler le différend. Dans d'autres cas, l'O.C.D.E. peut prendre l'initiative et effectuer une enquête sur les difficultés qui se présentent dans un domaine particulier. L'Organisation peut également procéder à une enquête systématique, pays par pays, sur les règlements appliqués en une matière déterminée. Dans le dernier cas, l'enquête aboutira vraisemblablement à la mise au point de règles normalisées que tous les pays Membres seraient instamment invités à adopter.

Da ans le passé, les actions particulières entreprises par l'O.E.C.E. et l'O.C.D.E. ont porté principalement sur les règlements du second groupe c'est-à-dire, sur ceux qui ne concernent pas directement les échanges. Les exemples suivants permettent de se rendre compte de la nature des travaux de l'Organisation dans ce domaine.

- Le Conseil a recommandé aux Gouvernements des pays Membres d'harmoniser et de simplifier dans toute la mesure possible les procédures d'étiquetage et d'enregistrement des produits pharmaceutiques et de tendre vers la reconnaissance réciproque des enregistrements nationaux; à cet effet, il a publié pour l'étiquetage et l'enregistrement, un ensemble de normes dont il a recommandé l'adoption par les pays Membres.

- Le Conseil a fixé un régime pour l'application de normes internationales de qualité à certains produits agricoles tels que pommes, poires et tomates.

- Le Conseil a adopté une résolution fixant des normes pour l'application et le contrôle des réglementations nationales de sécurité concernant les bouteilles à gaz soudées.

L'O.C.D.E. a récemment intensifié ses travaux portant sur les règlements administratifs qui sont en relation directe avec les échanges et en particulier sur ceux qui concernent les formalités douanières (y compris les procédures d'évaluation et classification des marchandises) et les procédures d'achat par l'État.

A l'occasion du nouvel exercice de négociations commerciales qui va se tenir dans le cadre de la loi américaine sur le développement des échanges (Trade Expansion Act), le lien étroit entre les problèmes que posent les règlements administratifs, d'une part, et la réduction des obstacles aux échanges, d'autre part, est apparu plus évident. Certains grands pays ont fait savoir qu'ils n'étaient plus disposés à prendre part à des négociations tarifaires si les concessions qu'ils pourraient obtenir risquaient d'être annulées par les effets protectionnistes de règlements administratifs. 


\section{ADAPTATION}
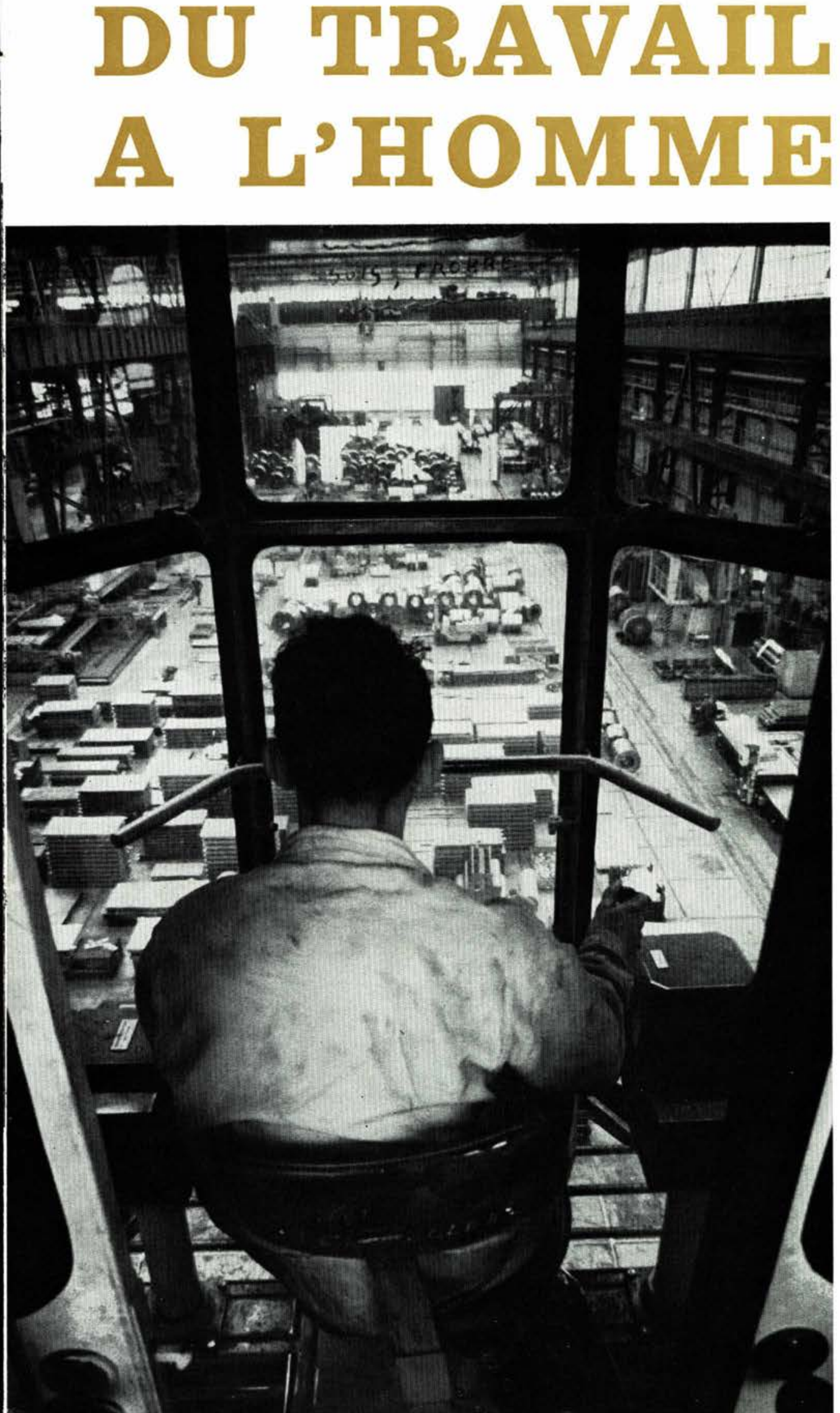

On peut adapter le travail à l'homme, mais cette adaptation est impossible si l'équipement est mal conçu ou si l'homme est obligé de travailler dans un état de tension.

«On recherche, ouvrier tourneur. Taille 1,35 m, largeur d'épaules $60 \mathrm{~cm}$, envergure $2,40 \mathrm{~m}$. " Tel serait, selon le chargé de cours en ergonomie du College of Aeronautics de Cranfield, en Angleterre, l'ouvrier idéal pour servir les tours de type courant.

Il est bien évident qu'on ne peut trouver un homme répondant à ces conditions. Il faut donc revoir la conception des tours, pour permettre aux ouvriers de les faire fonctionner.

La réussite d'une fabrication dépend de l'effort conjugué de la machine et de l'opérateur. Si l'un ou l'autre est soumis à des tensions anormales, le rendement en souffre. La machine peut être adaptée aux exigences humaines, mais si l'homme est obligé de travailler dans des conditions entraînant un état de tension ou ne correspondant pas exactement à son type de réactivité, son rendement diminuera et par suite celui de la machine.

L'ergonomie est une discipline où convergent diverses sciences correspondant à l'utilisation, par l'ouvrier, de son intelligence, de ses réflexes et de sa structure physique, dans la conduite de la machine. Faire de l'ergonomie, c'est en fait :

- diminuer la fatigue,

- accroitre la sécurité,

- obtenir des réactions plus rapides sans accroître la tension de l'ouvrier, 
- améliorer la qualité du travail,

- accélérer la formation du personnel.

L'ergonomie implique l'amélioration des conditions de travail, en tenant compte de facteurs tels que le confort physique, l'éclairage, le chauffage, l'atténuation du bruit, ainsi que des aspects psychologiques que constituent des commandes bien conçues et bien disposées et des cadrans indicateurs faciles à lire.

Dans le monde entier, des équipes de physiologistes, de phychologues, de médecins du travail, de dirigeants d'entreprises, d'ingénieurs, de syndicalistes et d'ouvriers s'efforcent d'adapter le travail à l'homme, améliorant ainsi les conditions de travail et le rendement. Depuis 1956, l'O.E.C.E., puis l'O.C.D.E., ont contribué, dans le cadre de leurs recherches sur les facteurs de base affectant la croissance économique, à centraliser et à diffuser largement les données de l'ergonomie, en organisant des conférences d'experts et des sessions d'étude. Trois rapports ont déjà été publiés sur l'adaptation du travail à l'homme. Un quatrième doit paraître prochainement*.

Ces photographies qui viennent d'Europe et l'Amérique du Nord donnent une idée des problèmes qu'étudient les ergonomistes, et de certaines des solutions adoptées.

- Adaptation du travail à l'homme (Recherches américaines et européennes sur les conditions de travail dans l'industrie. O.E.C.E. 1958).

- Adaptation du travail à l'homme (Conférence internationale de Zurich. O.E.C.E. 1959).

- A propos de l'adaptation du travail à l'homme (Collection Formation syndicale. O.E.C.E. 1961).

- Session d'étude surl'ergonomie pour les ingénieurs (O.C.D.E. 1963).
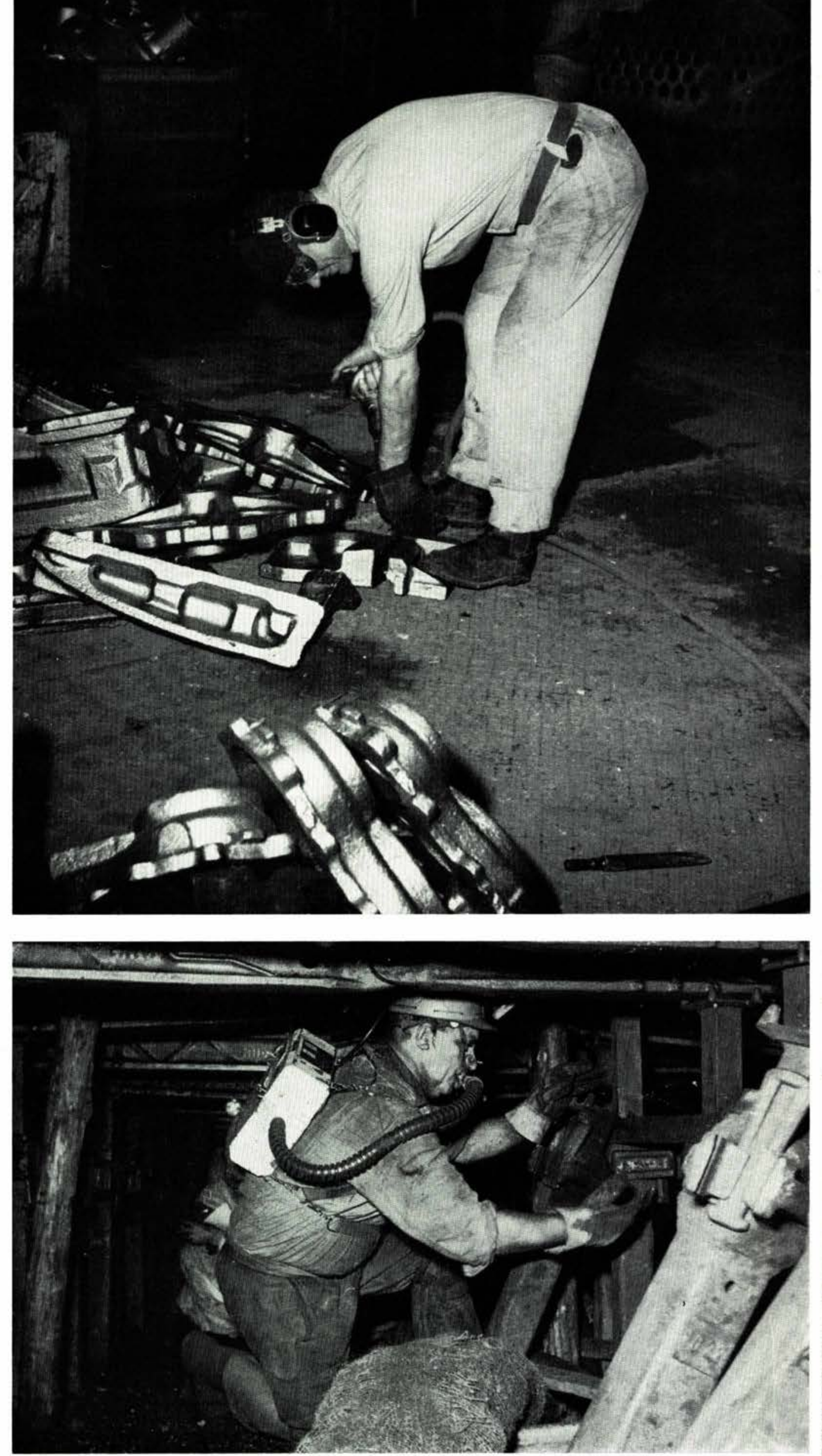
4 Contrôle des produits moulés dans une usine sidérurgique; un casque protège l'ouvrier du vacarme de l'usine (Régie Nationale des Usines Renault, France).

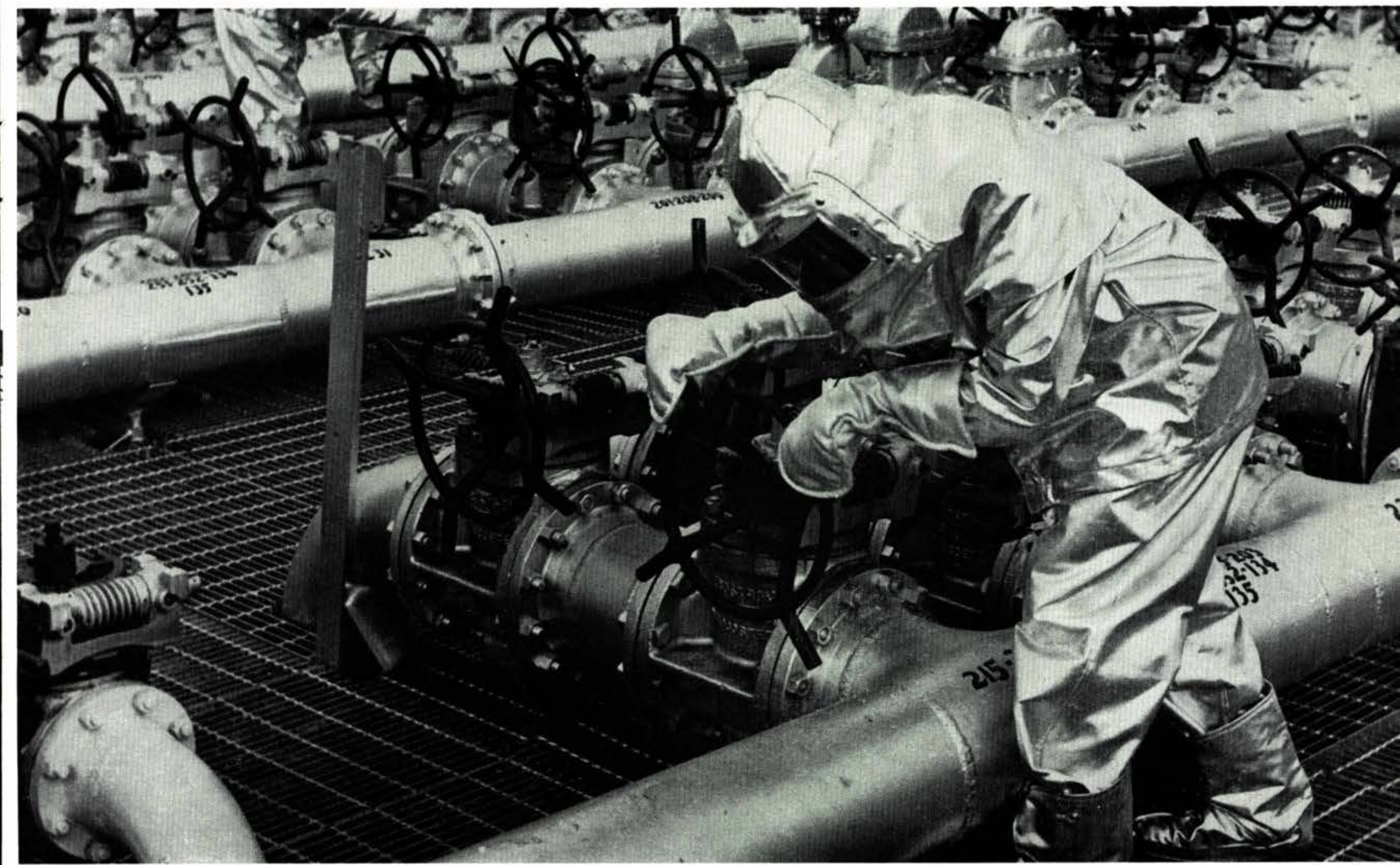

$\Delta$

PROTECTION

Dans une raffinerie de pétrole, les ouvriers portent des vêtements de protection en tissu aluminisé (Minnesota Mining and Manufacturing Company, États-Unis).

Placé derrière un écran de verre et de métal qui le protège des fumées et des poussières, un ouvrier de l'aéronautique garde le libre usage de ses mains (Aéroport d'Orly, France).

L'appareil respiratoire que porte ce mineur lui évite d'absorber des poussières de charbon (Max-PlanckInstitut für Arbeitsphysiologie, Allemagne).

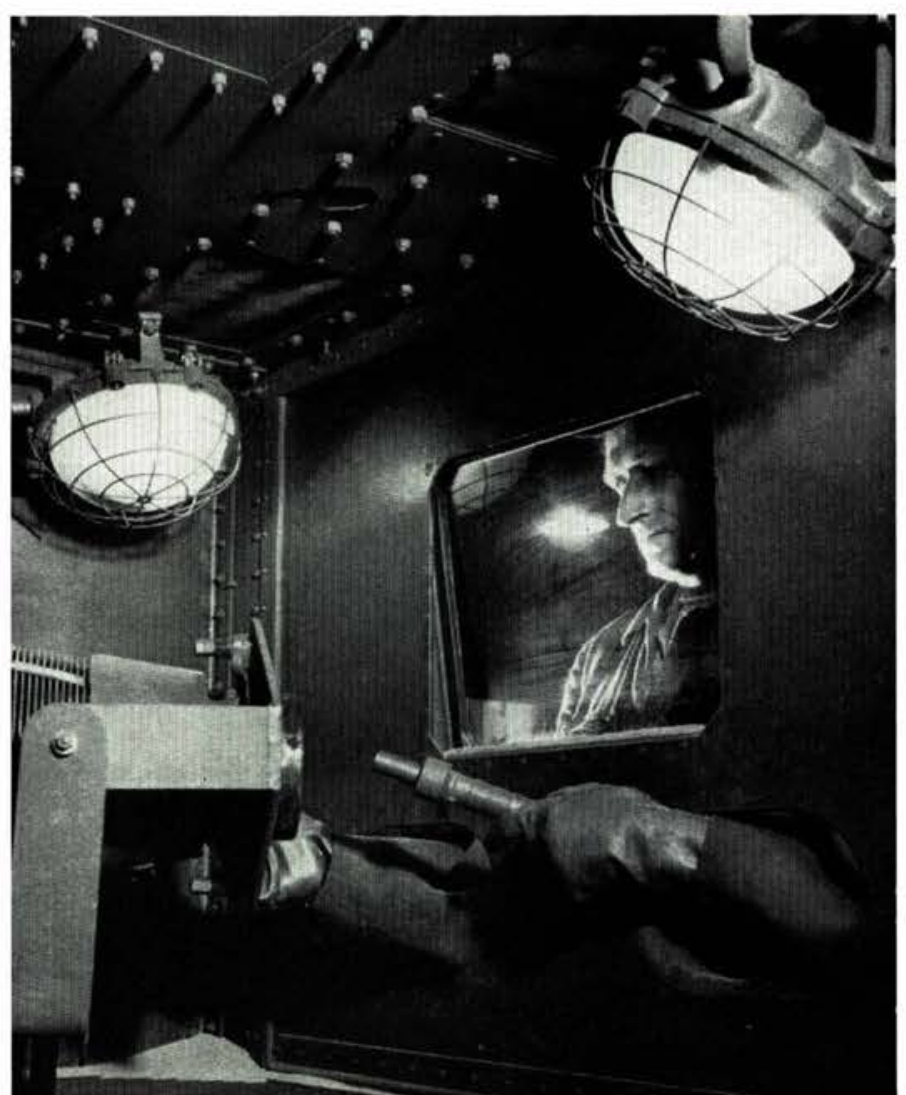




\section{LE PROBLEME DE}

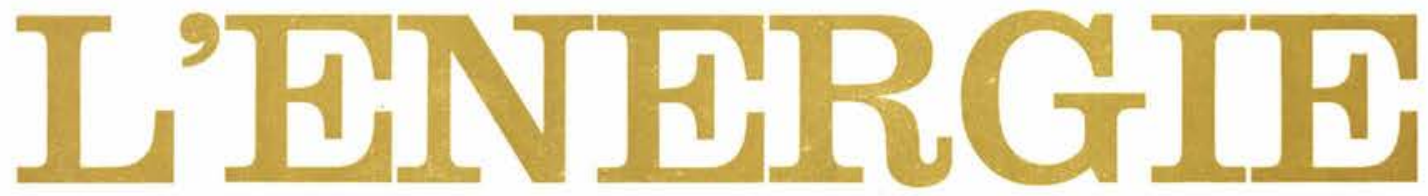

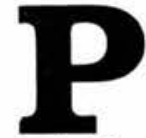

endant les années qui ont immédiatement suivi la deuxième guerre mondiale et jusque bien après 1950, la crainte de voir une pénurie d'énergie s'installer en Europe occidentale a dominé les discussions des personnes responsables dans les Gouvernements. Cependant, vers 1957, cette crainte s'est trouvée subitement dissipée et depuis lors elle a fait place à une nouvelle inquiétude suscitée par les problèmes résultant d'une concurrence de plus en plus acharnée entre différentes formes d'énergie. Comment ce retournement de la situation a-t-il pu se produire? Quelles en sont les raisons?

\section{LA PSYCHOLOGIE DE LA PÉNURIE}

Dans les années qui ont suivi immédiatement la deuxième guerre mondiale, le monde et particulièrement l'Europe occidentale ont souffert incontestablement d'une pénurie d'énergie. Jusqu'en 1940 , les sources indigènes avaient pu couvrir en très grande partie les besoins de l'Europe, mais au cours du conflit les industries productrices d'énergie ont subi de très graves dommages, du fait de destructions directes, de l'usure prématurée due aux efforts intenses déployés pendant la guerre, et aussi du défaut d'entretien ou d'investissements réalisés à temps. A partir de 1948, de nombreuses réparations ont pu être effectuées, de nouvelles usines sont entrées en service, mais c'est seulement vers 1951 que la production indigène a rattrapé et dépassé le niveau d'avant la guerre. Néanmoins, dès cette époque, elle aurait pu être très supérieure à ce niveau pour satisfaire les besoins d'une économie en voie de développement.

L'un des principaux avantages de l'aide Marshall est qu'elle a permis à l'Europe occidentale de reconstruire ses industries énergétiques et d'en accroître la production. Mais cette aide a eu également un autre effet : grâce à elle, l'Europe a pu surmonter la pénurie de dollars qui était l'un des plus graves obstacles aux importations de charbon et de pétrole dont elle avait besoin pour combler le fossé entre ses propres ressources encore déficientes et ses besoins rapidement croissants en énergie.

L'aide Marshall a puissamment contribué à la reconstruction et au développement des charbonnages et des cokeries, à la construction de logements pour les familles de mineurs, à la remise en état des centrales électriques et des usines à gaz et à la construction de nouvelles centrales hydroélectriques et thermiques. Les voies navigables intérieures, les ports de mer et les autres moyens de transport ont été rééquipés et développés. L'industrie pétrolière a pu construire en Europe une capacité de raffinage capable de satisfaire, principalement à partir de brut importé, les besoins croissants du continent en produits raffinés. Ainsi, l'aide Marshall n'a pas seulement aidé l'Europe à s'équiper pour pouvoir économiser des dollars : elle lui a également permis d'accroître sa capacité de production, et ainsi de nourrir ses exportations et de gagner les devises nécessaires à un nouveau développement économique. Comme on l'avait voulu, l'aide Marshall a agi comme un catalyseur : son effet s'est très largement multiplié par les efforts nationaux qu'elle avait suscités.

Cependant, à mesure que la reconstruction avançait, il devenait urgent de relever la producti- 

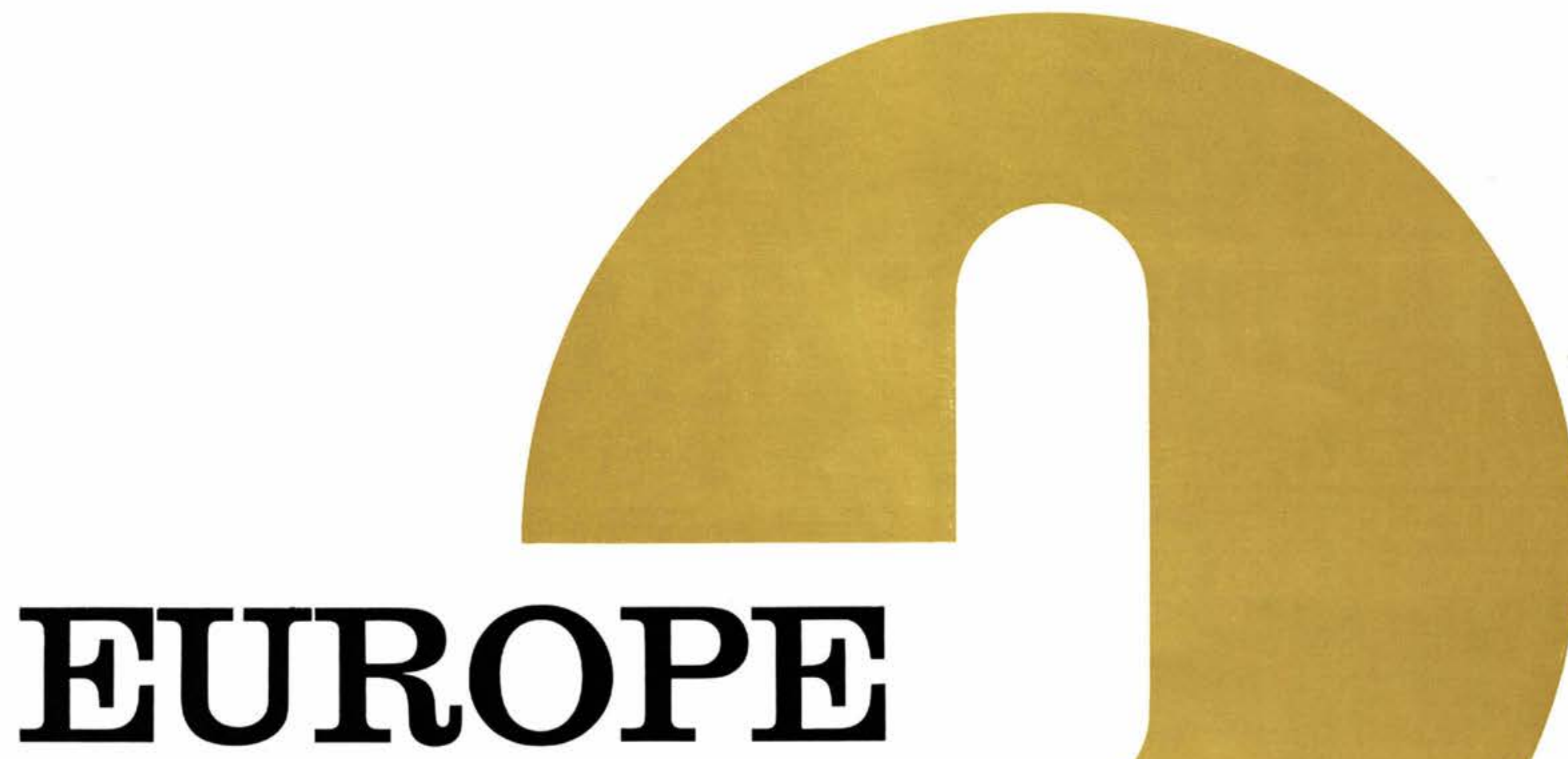

vité de l'industrie, de l'agriculture et du secteur des services. Dans une mesure croissante, l'Europe a pris conscience du fait qu'elle était de plus en plus tributaire de l'importation pour les approvisionnements en énergie indispensable à la réalisation des tâches qu'elle s'était fixées, et notamment à l'élévation du niveau de vie de ses populations. Depuis 1930, époque où l'Europe a perdu son autonomie énergétique, passant du groupe des exportateurs potentiels d'énergie à celui des importateurs permanents, ce fait était passé inaperçu. Le problème se posait maintenant dans le contexte d'une économie mondiale et dans des conditions politiques qui rendaient infiniment plus menaçants ses différents aspects. En 1951, la crise de Corée a produit l'effet d'un choc. Loin de se limiter au domaine de l'énergie, la pénurie s'est étendue à de nombreuses matières premières. Les frets maritimes, qui entrent pour une large part dans le coût du charbon et du pétrole importés, sont montés en flèche. Puis, alors que cette crise s'apaisait, une nouvelle crise a éclaté en 1956-57 avec la fermeture du canal de Suez. Certes, l'Europe ne s'est pas trouvée à cette époque coupée de l'accès au pétrole de plus en plus indispensable à son économie, mais les changements inévitables intervenus dans ses sources d'approvisionnement et dans les courants de transport ont engendré des pénuries temporaires et déclenché une nouvelle hausse considérable du coût de l'énergie importée, qu'il s'agisse de pétrole ou de charbon.

L'Europe a ainsi été amenée à réfléchir sérieusement à la vulnérabilité de ses approvisionnements énergétiques, en l'absence desquels elle ne pourrait

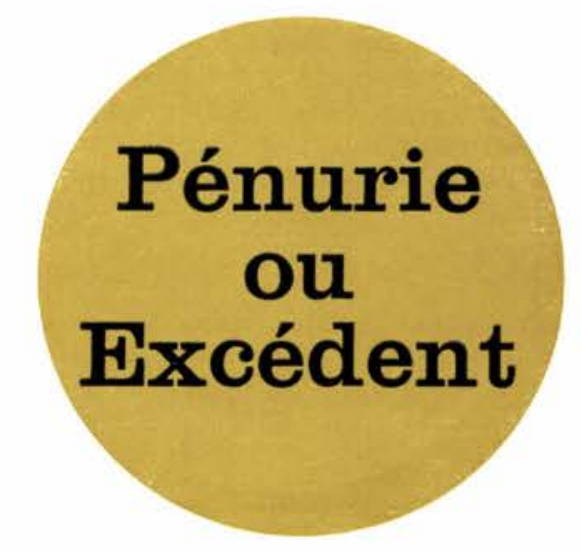

Le présent article a été écrit par

Jan A. Van den Heuvel

Chef de la Division de l'Énergie,

Direction de l'Industrie de l'O.C.D.E. 


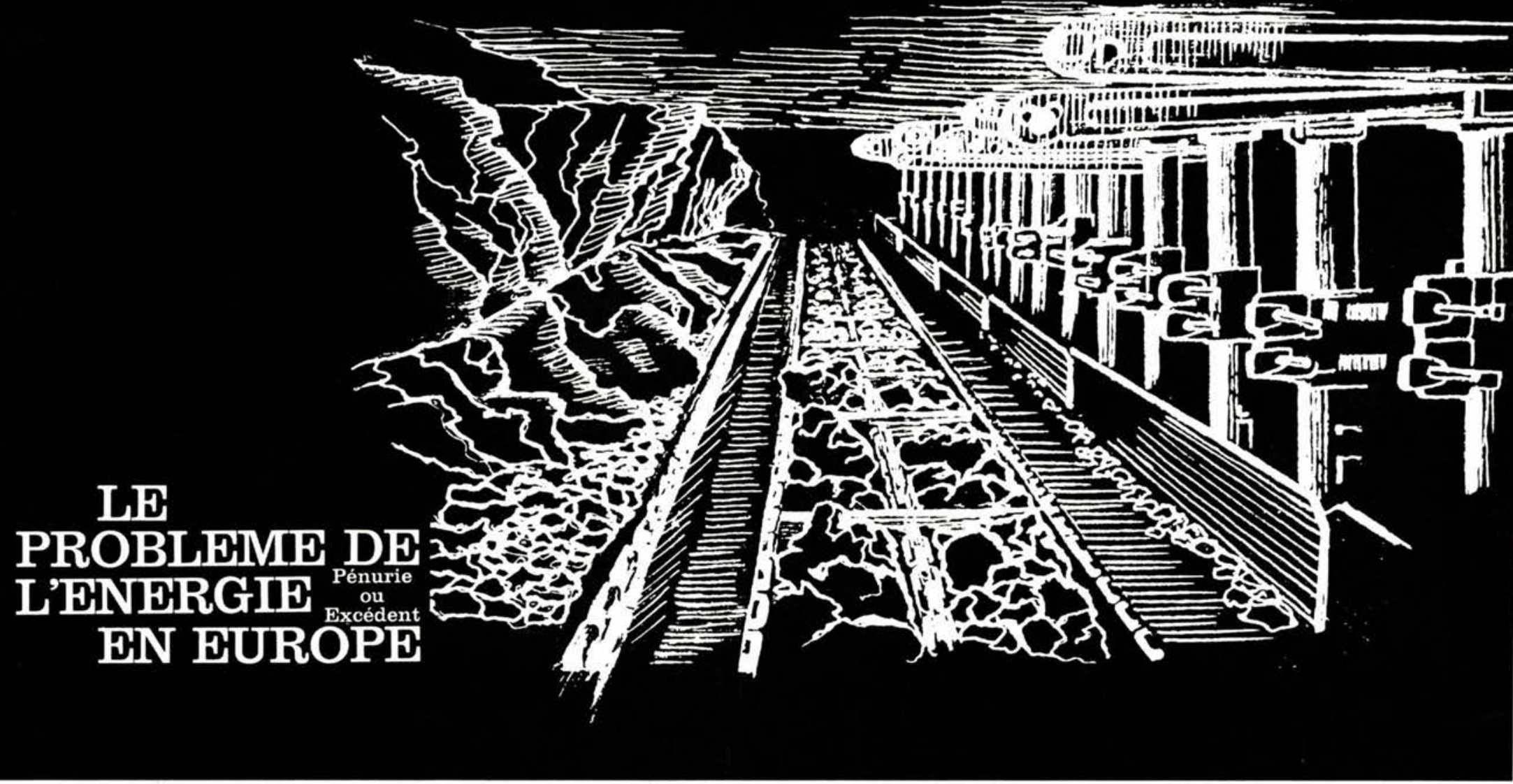

demeurer la grande région industrielle qu'elle est, et encore moins recouvrer la situation qu'elle occupait au début du siècle. Portant ses regards vers l'avenir, elle a constaté que ses besoins allaient très vraisemblablement dépasser de loin sa capacité de production. Elle a conclu qu'il lui faudrait déployer de vigoureux efforts pour développer sa production intérieure et la porter à un niveau compatible avec le maintien d'un coût global économiquement justifié pour son énergie. La position existante ainsi que les perspectives d'aveni de sa balance générale des paiements ont fortement influé sur son attitude à cet égard.

\section{LES RAISONS D'UN NOUVEL OPTIMISME}

Pendant cette période, et souvent pour des raisons étroitement liées à celles qui incitaient à considérer la réalité avec prudence, voire avec pessimisme, une nouvelle tendance est apparue. Elle repose sur un certain nombre d'éléments qui peuvent se résumer comme suit

\section{- Utilisation plus rationnelle de l'énergie}

La part des combustibles liquides dans la consommation totale d'énergie a augmenté beaucoup plus rapidement qu'on ne l'avait prévu. De plus en plus, ces produits se substituent aux combustibles solides. Dans bien des secteurs de la consommation, leur emploi permet d'améliorer les rendements et d'accroître la production par unité consommée. D'autre part, de nouveaux procédés de production ont été adoptés par l'industrie sidérurgique et les méthodes de production traditionnelles ont été sensiblement améliorées. Comme cette industrie est l'un des grands consommateurs d'énergie, les économies qui en sont résultées dans l'utilisation ont eu des conséquences importantes sur les besoins globaux en énergie. La même considération s'applique à l'industrie de l'électricité, qui est aussi un très important consommateur d'énergie primaire. Grâce aux améliorations introduites dans les types traditionnels de centrales, grâce en particulier à la mise en service de groupes beaucoup plus puissants, on a pu abaisser progressivement la quantité d'énergie primaire nécessaire pour produire un kilowatt-heure.

\section{- Amélioration des termes de l'échange en Europe}

La balance des paiements de l'Europe n'a cessé de s'améliorer. La pénurie chronique de dollars a disparu dans la plupart des pays, et les craintes qu'elle suscitait de la part de ceux qui voyaient s'aggraver leur dépendance à l'égard des importations ont peu à peu disparu.

\section{- Accroissement et diversification des} ressources énergétiques dans les régions exportatrices

Stimulée par les pénuries qu'elle avait connues dans les premières années postérieures à la guerre, l'industrie pétrolière internationale a fait un très gros effort pour développer et diversifier sa production de brut. Alors qu'en 1950, les réserves prouvées de pétrole du monde non communiste s'élevaient à environ 14 milliards de tonnes, soit 23 années de consommation au taux d'alors, en 1960 ces réserves atteignaient environ 41 milliards de tonnes, soit 40 années de consommation au taux de 1960, quí représentait le double de celle de 1950. De nouvelles zones de production pétrolière de grande importance, comme le Sahara et la Libye, sont apparues qui, du point de vue des transports, présentent de gros avantages pour l'Europe occidentale. La Russie soviétique a pris place sur le marché comme un grand exportateur de pétrole; elle offre ses produits à des prix remarquablement bas. Et les États-Unis, en restreignant leurs importations de divers combustibles liquides, ont détourné les excédents mondiaux de pétrole vers le marché européen, qui est le plus gros importateur du monde. Mais l'abondance de pétrole n'est pas la seule raison qui justifie un optimisme nouveau : d'importantes découvertes de gaz naturel ont été faites dans 
grands bouleversements économiques ou politiques, on peut maintenant tenir pour assuré que la situation excédentaire actuelle persistera jusqu'à cette époque; mais il est indispensable de déterminer comment la situation évoluera après 1975. Les besoins mondiaux en énergie augmentent rapidement et les ressources actuellement connues seront mises à forte contribution pour faire face à la demande. L'équipement de nouvelles sources d'énergie exige la plupart du temps de nombreuses années et des investissements énormes, qui ne peuvent être récupérés que sur de longues périodes. La marge entre les situations de pénurie et d'excédent est parfois étonnamment étroite; elle dépend en grande partie de facteurs psychologiques. De toute évidence, le monde occidental sera bien inspiré de suivre attentivement l'évolution de sa situation.

\section{LA CONSOMMATION DE L'ENERGIE PRIMAIRE DANS LES PAYS MEMBRES EUROPEENS DE L'OCDE}
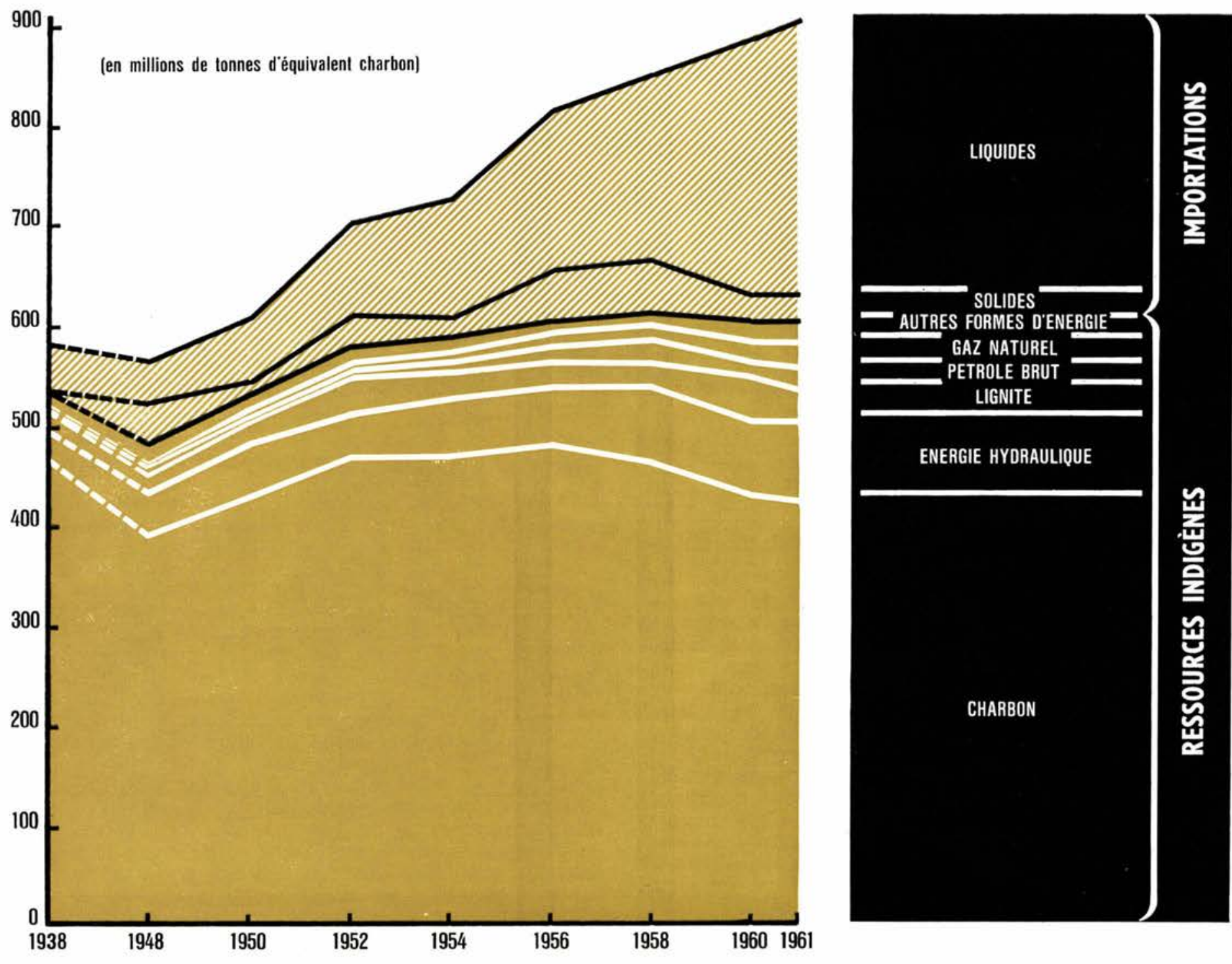

$=$

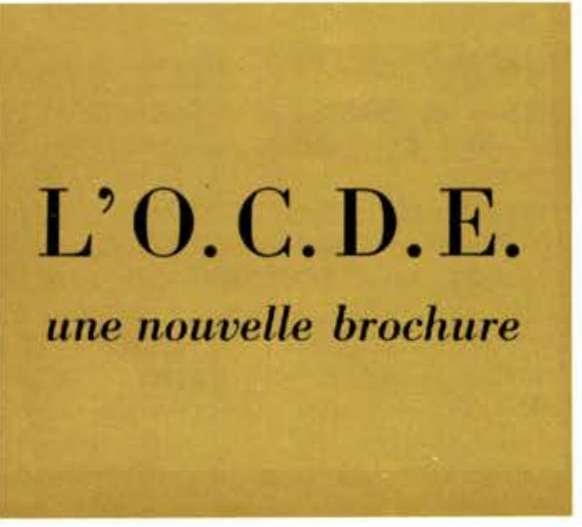

Cette brochure contient les informations essentielles sur l'historique, les buts et tâches, la structure et les fonctions de l'Organisation; on y trouve, en outre, dans une partie documentaire, la Convention relative à l'O.C.D.E., le Règlement de procédure, l'Accord monétaire européen, les Codes de la libération des mouvements de capitaux et des opérations invisibles courantes, ainsi que les Statuts de l'Agence Européenne pour l'Energie Nucléaire.

Cette brochure, qui vient d'être publiée, est distribuée gratuitement sur demande adressée au Service d'Information de l'O. C. D. E., 2, rue André Pascal, Paris XVI ${ }^{\mathrm{e}}$, ou aux Dépositaires des publications de l'O.C.D.E. 


\section{OU OBTENIR LES PUBLICATIONS DE L'O.C.D.E.}

AFRIQUE DU SUD

Van Schaik's Book Store Ltd., Church Street, Pretoria.

\section{ALLEMAGNE}

Deutscher Bundes-Verlag

Postfach 9380, 53 BoNN

Sous-dépositaires : BerLIN : Elwert \&

Meurer.

MUNICH : Hueber.

HAMBourg : Reuter-Klöckner;

et dans les principales librairies d'Allemagne.

\section{ARGENTINE}

Editorial Sudamericana S.A., Alsina 500, Buenos Aires.

\section{AUSTRALIE}

B.C.N. Agencies Pty. Ltd., 62 Wellington Parade, East Melbourne, C.2.

\section{AUTRICHE}

Gerold \& Co., Graben 31, VIENNE 1.

Sous-dépositaire :

Buchhandlung Jos. A. Kienreich,

Sackstrasse 6, Graz.

\section{BELGIQUE}

N.V. Standaard-Boekhandel.

Huidevettersstraat 57, ANVERS.

Librairie des Sciences (R. Stoops),

76-78, Coudenberg, BruXelles.

\section{BRÉSIL}

Livraria Agir Editôra,

Rua Mexico 98-B, Rio de Janeiro.

CANADA

Queen's Printer - Imprimeur de la Reine, OTTAWA.

Les commandes sont payables d'avance.

CUBA

La Casa Belga, O'Reilly 455, La Havane.

DANEMARK

Ejnar Munksgaard Forlag, Nörregade 6, Copenhague.

\section{ESPAGNE}

Mundi Prensa, Castelló 37, Madrid. Libreria Bosch, Ronda Universidad II, BARCELONE.
ÉTATS-UNIS D'AMÉRIQUE

O.E.C.D. Regional Office,

Suite 1223. 1346 Connecticut Ave N.W., WASHINGTON 6, D.C.

FINLANDE

Akateeminen Kirjakauppa, Keskuskatu 2, HELSINKI.

\section{FRANCE}

Presses Universitaires de France, 108 , boulevard Saint-Germain, Paris $\left(6^{\mathrm{e}}\right)$.

\section{GRÈCE}

Librairie Kauffmann, 21, rue du Stade, ATHÈNES.

\section{INDE}

International Book House Ltd., 9 Ash Lane, Mahatma Gandhi Road, BOMBAY I.

Oxford Book and Stationery Co.,

New Delhi, Scindia House.

Calcutta, 17 Park Street.

\section{IRLANDE}

Eason \& Son. 40-41 Lower O'Connell Street, Dublin.

\section{ISLANDE}

Snæbjörn Jónsson \& Co, h.f.,

Hafnarstræti 9 ,

P.O. Box 1131, REYKJAVIK.

\section{ISRAEL}

Blumstein's Bookstores Ltd., 35 Allenby Road, and 48 Nahlath Benjamin St., Tel-Aviv.

\section{ITALIE}

Libreria Commissionaria Sansoni

Via Gino Capponi 26, Florence.

Via Paolo Mercuri 19/B. Rome.

Corso Cavour 39, BARI.

P.E.M., Corso di Porta Romana 87/B, Milan.

Sous-dépositaires :

GÊNES : Libreria Di Stefano.

Milan : Libreria Hœpli.

NAPLES : Libreria L. Cappelli.

PADOUE : Libreria Zannoni.

Palerme : Libreria C. Cicala Inguaggiato.

RoME : Libreria Hœpli; Libreria Tombolini.

TURIN : Libreria Lattes.
JAPON

Maruzen Company Ltd., 6 Tori Nichome Nihonbashi, Toкyo.

LUXEMBOURG

Librairie Paul Bruck, 33, Grand'Rue, LUXEMBOURG.

MAROC

B.E.P.I., 8, r. Michaux-Bellaire, RABAT.

NIGERIA

University Bookshop Nigeria Ltd., University College, IBADAN.

\section{NORVÈGE}

A/S Bokhjornet, Lille Grensen 7, OsLo.

PAKISTAN

Mirza Book Agency,

9-A Shah Alam Market, LAHORE.

\section{PAYS-BAS}

Grossiste :

Meulenhoff \& Co., N.V. Importeurs, Beulingstr., 2, AMSTERDAM.

Détaillant :

W.P. Van Stockum \& Zoon,

Buitenhof 36, LA HAYE.

\section{PORTUGAL}

Livraria Portugal, Rua do Carmo 70 , LISBONNE.

\section{ROYAUME-UNI ET}

COLONIES DE LA COURONNE

H.M. Stationery Office, P.O. Box 569,

LONDRES, S.E.I.

Annexes à : Edinbourg, Birmingham, Bristol, Manchester, Cardiff, Belfast.

SUÈDE

Fritzes. Kungl. Hovbokhandel, Fredsgatan 2, Sтоскноцм 16.

\section{SUISSE}

Librairie Payot,

40, rue du Marché, Genìve

et à Lausanne, Neuchatel, Vevey,

Montreux, Berne, Bale et Zurich.

TURQUIE

Librairie Hachette, 469 Istiklal Caddesi, Beyoglu, Istanbul.

\section{YOUGOSLAVIE}

Jugoslovenska Knjiga, Marsala Tita, 23, P.O.B. 36, Belgrade.

Les commandes provenant de pays où l'O.C.D.E. n'a pas encore désigné de dépositaire peuvent être adressées à : O.C.D.E., Bureau des Publications, 2, rue André-Pascal, Paris (16 ${ }^{\mathrm{e}}$ 


\section{Organisation de Coopération et de Développement Economiques}

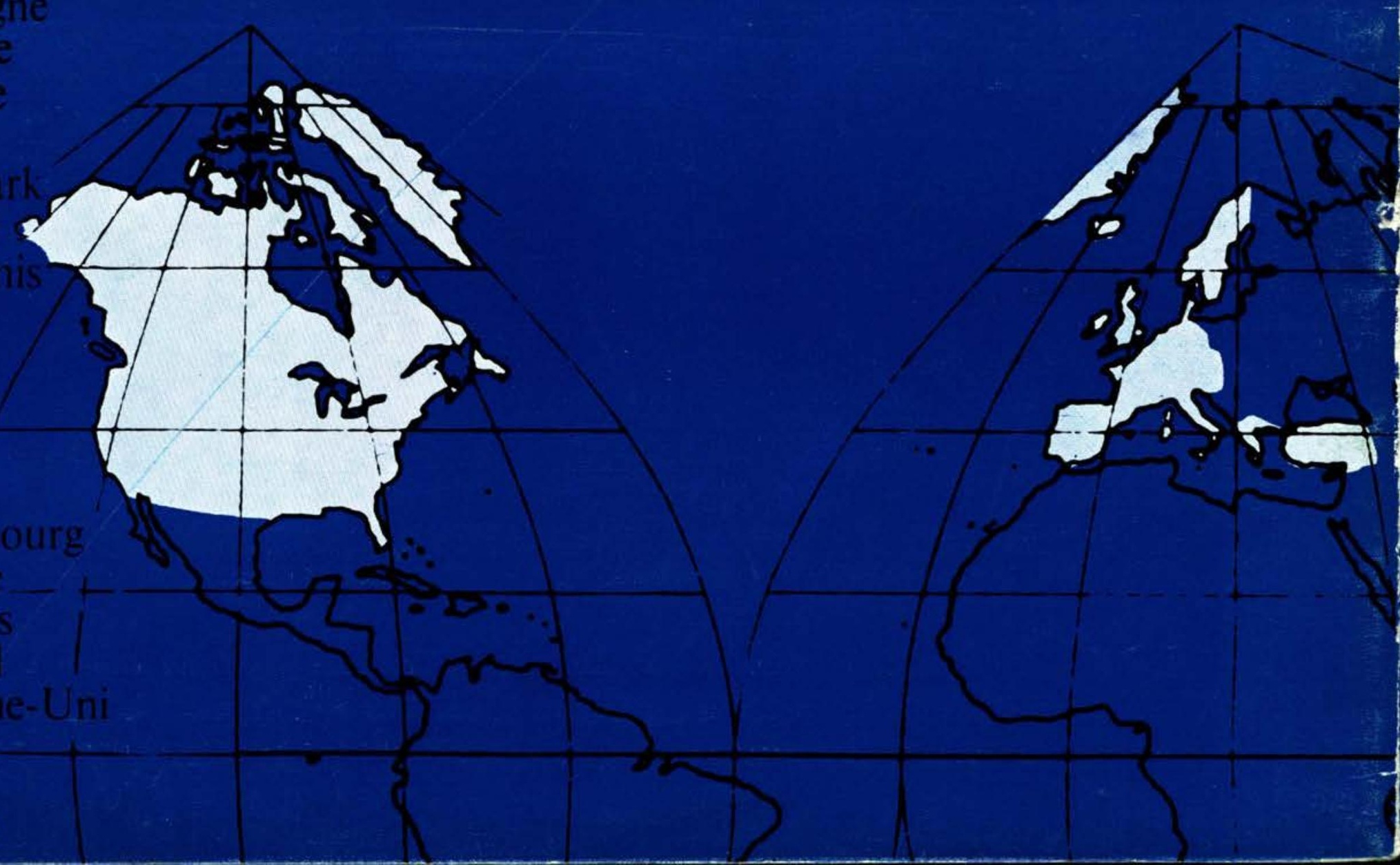

